\title{
Far-infrared spectroscopy across the asymmetric bipolar outflows from Cepheus A and L 1448^
}

\author{
D. Froebrich ${ }^{1}$, M. D. Smith ${ }^{2}$, and J. Eislöffel ${ }^{1}$ \\ 1 Thüringer Landessternwarte Tautenburg, Sternwarte 5, 07778 Tautenburg, Germany \\ 2 Armagh Observatory, College Hill, Armagh BT61 9DG, Northern Ireland
}

Received 9 August 2001 / Accepted 17 January 2002

\begin{abstract}
Bipolar outflows are driven from protostars within molecular cores. They drive into molecular clouds, generating shock waves whose molecular emission lines have been observed in the infrared with ISO. We present spectroscopic data for seven locations within two asymmetric outflows, Cepheus A and L 1448, in order to test the shock physics and shock dynamics. Here, we simultaneously interpret the $\mathrm{CO}$ and $\mathrm{H}_{2}$ data sets which are generated by shocked gas, radiating at temperatures from 300 to $2000 \mathrm{~K}$. We find that large-scale spatial variations in the excitation are absent across both outflows and that the excitation is low everywhere. Planar shock models are inconsistent with the data sets. Models with configurations or ensembles of shocks, in the form of bow shocks or supersonic turbulence, are consistent. This solves the previously reported problem that the CO abundances were anomalously high. Cool gas is dominant, from which we infer bow shocks with flanks more extended than for paraboloids. As a consequence, the atomic oxygen abundances must be quite low. $J$-type bow models require implausibly long wings. $C$-type physics is thus favoured. The density and the ratio of molecules to atoms are constrained by the $\mathrm{CO} / \mathrm{H}_{2}$ flux levels as well as the $\mathrm{H}_{2}$ vibrational level distributions. Other $C$-shock parameters, such as the magnetic field strength, ion fraction and speed, are not tightly constrained. The total shock powers are derived and are comparable to the mechanical outflow luminosities for both outflows, consistent with the outflows being momentum-driven.
\end{abstract}

Key words. shock waves - molecular processes - ISM: jets and outflows - ISM: kinematics and dynamics ISM: molecules - stars: mass-loss

\section{Introduction}

Bipolar outflows plough into the environments of their driving protostars, generating bow-shaped shock structures. On scales comparable to the parental molecular cloud, they can be studied in the infrared even when the protostar itself is still hidden deep within a dense core. In order to learn about the energetics and evolution of the protostar and the star forming region, we first need to determine the shock physics and shock morphology. Ground-based telescopes have provided quality nearinfrared spectral data for many outflows (e.g. Fernandes \& Brand 1998). The ISO satellite, however, offered a unique opportunity to gain spectral data on the shocked molecular gas which radiates profusely from the near- to the

Send offprint requests to: D. Froebrich, e-mail: frobich@tls-tautenburg.de

* Based on observations with ISO, an ESA project with instruments funded by ESA Member States (especially the PI countries: France, Germany, the Netherlands and the United Kingdom) and with the participation of ISAS and NASA. far-infrared. Here we evaluate two outflows which were observed at several locations, Cepheus A and L 1448.

The two opposite lobes of each of these outflows are highly asymmetric, one appearing quite spatially turbulent and the other containing ordered shock structures. The Cepheus A outflow has at least one early B star at its source, with a high mass outflow and mechanical luminosity of $\sim 4 \times 10^{-4} M_{\odot}$ and $\sim 60 L_{\odot}$ (e.g. Narayanan \& Walker 1996). The L 1448 outflow, on the contrary, has an order of magnitude lower estimated mass outflow rate and a mechanical power of $\sim 2 \times 10^{-5} M_{\odot}$ and $\sim 0.3 L_{\odot}$ (O`Linger et al. 1999). Apart from the L $1448 \mathrm{C}$ outflow, which originates from a Class 0 protostar, several other flows are observed to its north, which also emanate from deeply embedded young stellar objects (e.g. Eislöffel 2000).

Specific sets of ISO data for Cepheus A East and West have been investigated by van den Ancker et al. (2000) and Wright et al. (1996), respectively. Uniform components and planar shocks were tested and temperatures for the low-lying $\mathrm{H}_{2}$ rotational levels of $\sim 700 \mathrm{~K}$ were found. A full set of ISO data has been analysed for L 1448 by 


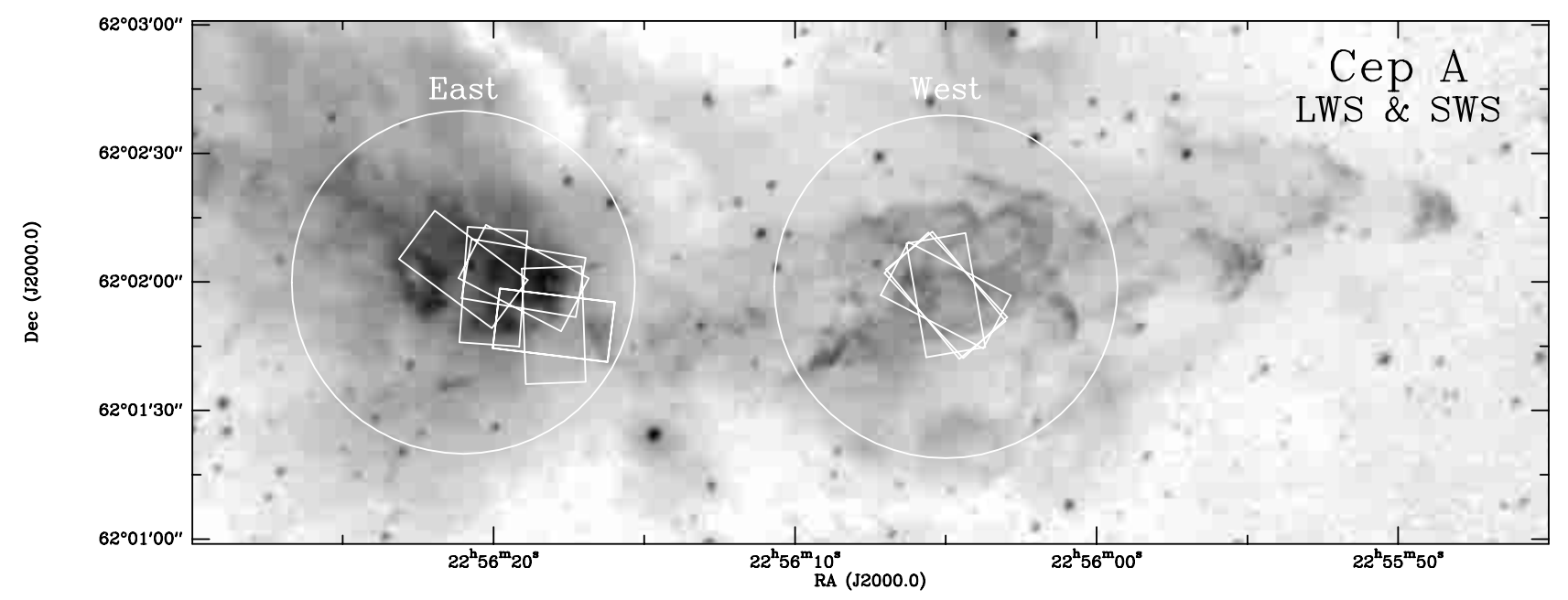

Fig. 1. Positions of apertures of SWS and LWS observations in Cepheus A superimposed on an image in the 1-0 S(1) line of $\mathrm{H}_{2}$ at $2.12 \mu \mathrm{m}$.

Nisini et al. (1999) and Nisini et al. (2000), who concluded a best fit temperature to both the $\mathrm{CO}$ and $\mathrm{H}_{2}$ of $\sim 1300 \mathrm{~K}$. Here, we introduce and apply detailed shock models to the full ISO data sets. We thus find that we can differentiate between several shock models, involving the chemistry (e.g. oxygen abundance), the physics (ambipolar diffusion) and the geometry (degree of curvature).

We present the observational results in Sect. 3. We detect gas at temperatures of $300-2000 \mathrm{~K}$ and summarise the models which can generate this excitation range in Sect. 4. Strong non-dissociative $J$-shocks heat gas to above $10000 \mathrm{~K}$. Hence, we 'see' these shocks only when the gas has cooled, usually making them inefficient infrared radiators. $C$-shocks heat the gas directly to temperatures under $5000 \mathrm{~K}$ and emit strongly in the infrared. Bow shocks heat gas to a whole range of temperatures according to the location on the bow front. We use the accumulated set of models in Sect. 5 to interpret Cepheus A East. We have updated the old codes for $C$-shocks, $J$-shocks and both types of bow shock (e.g. Smith \& Brand 1990; Smith 1994b) by employing the $\mathrm{H}_{2}$ collisional rates according to Le Bourlot et al. (1999) and added vibrational $\mathrm{H}_{2} \mathrm{O}$ and $\mathrm{CO}$ cooling (Neufeld \& Kaufman 1993). In fact, we find no significant differences with the previous predictions such as used for ISO SWS observations of Cepheus A West (Smith 2000). Having established plausible shock models, we apply and adjust these to the other seven locations in Cepheus A West (Sect. 6) and L 1448 (Sect. 7). These results are then summarised and discussed in Sect. 8 .

\section{Observations and data reduction}

The spectra presented in this study were obtained using the ISO satellite (Kessler et al. 1996) with the SWS (de Graauw et al. 1996) and the LWS (Clegg et al. 1996) instruments. A log of the observations is provided in Table 1.

For Cep A, the positions of the spectrograph apertures are displayed in Fig. 1. Our position Cep A "East" includes data with object names Cep A IRS6A and Cep A (except

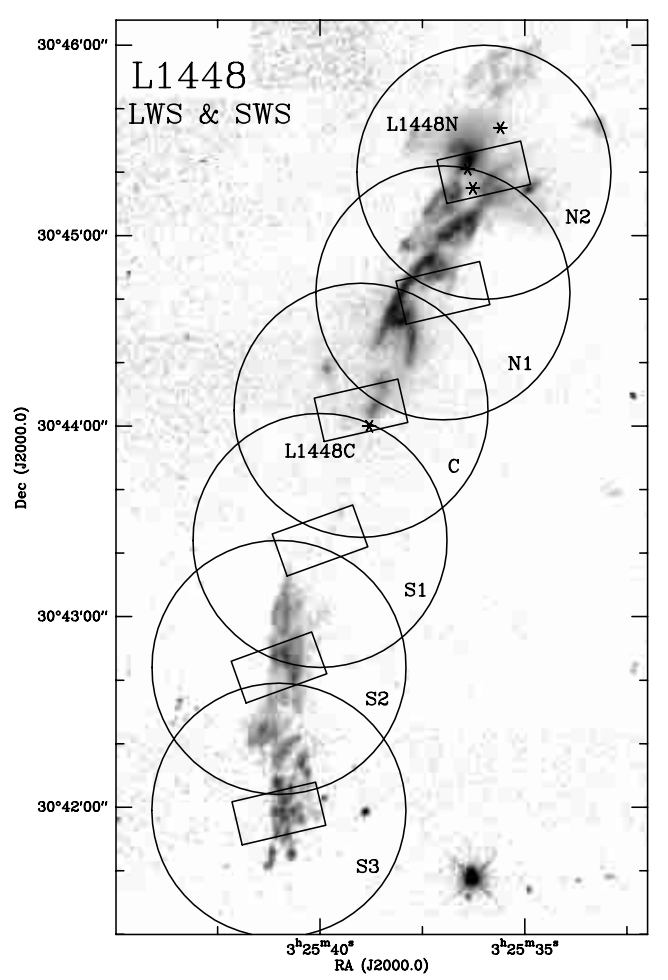

Fig. 2. Positions of apertures of SWS and LWS observations in $\mathrm{L} 1448$ superimposed on an image in the 1-0 S(1) line of $\mathrm{H}_{2}$ at $2.12 \mu \mathrm{m}$.

for observation 79201033) in the ISO data archive, while Cep A "West" assembles the Cep A (W), Cep A PK2 and the 79201033 data.

For L1448, the positions of the spectrograph apertures are shown in Fig. 2. Here, we have six different positions, which we name $\mathrm{N} 2, \mathrm{~N} 1$ in the northern lobe, $\mathrm{C}$ in the centre, and S1, S2, S3 in the southern lobe. They include the data with ISO names (see Table1) N and IRS3 for position N2, N2 for N1, L1448 ON for C, S1 for S1, S for S2, and S2 and SIO for S3. 
Table 1. Observation log for the Cepheus A and L 1448 data.

\begin{tabular}{lllllll}
\hline TDT & Object (ISO) & Position & $\alpha(\mathrm{J} 2000)$ & $\delta(\mathrm{J} 2000)$ & AOT \\
\hline 09605051 & Cep A PK2 & West & 225605 & +610157 & SWS02 \\
10101553 & Cep A PK2 & West & 225605 & +610159 & LWS01 \\
22000636 & Cep A IRS6A & East & 225619 & +610201 & SWS02 \\
22001001 & Cep A (W) & West & 225605 & +610157 & SWS02 \\
24800552 & Cep A PK2 & West & 225605 & +610157 & SWS07 \\
28301183 & Cep A & East & 225621 & +610202 & LWS01 \\
41400837 & Cep A & East & 225621 & +610203 & SWS06 \\
46500654 & Cep A PK2 & West & 225605 & +610159 & LWS04 \\
46500669 & Cep A PK2 & West & 225605 & +610159 & LWS01 \\
46500801 & Cep A (W) & West & 225605 & +610159 & LWS04 \\
46500802 & Cep A (W) & West & 225605 & +610159 & LWS01 \\
56300803 & Cep A & East & 225618 & +610150 & SWS07 \\
56300918 & Cep A & East & 225618 & +610148 & LWS04 \\
56301021 & Cep A & East & 225618 & +610150 & SWS02 \\
56600809 & Cep A IRS6A & East & 225619 & +610201 & SWS02 \\
56600810 & Cep A IRS6A & East & 225620 & +610159 & LWS02 \\
65701021 & Cep A & East & 225618 & +610150 & SWS02 \\
79201033 & Cep A & West & 225605 & +610157 & SWS02 \\
84300404 & Cep A IRS6A & East & 225620 & +610159 & SWS01 \\
65300362 & L 1448 N2 & N1 & 032537 & +304442 & LWS01 \\
65300363 & L 1448 S1 & S1 & 032540 & +304324 & LWS01 \\
65300364 & L1448 S2 & S3 & 032541 & +304159 & LWS01 \\
65300701 & L 1448 & C & 032539 & +304405 & LWS01 \\
65300702 & L 1448 N & N2 & 032536 & +304520 & LWS01 \\
65300703 & L 1448 S & S2 & 032541 & +304244 & LWS01 \\
81401066 & L 1448 ON & C & 032539 & +304405 & SWS02 \\
81401067 & L1448 IRS3 & N2 & 032536 & +304520 & SWS02 \\
81601568 & L 1448 N2 & N1 & 032537 & +304442 & SWS02 \\
81601569 & L 1448 SIO & S3 & 032541 & +304158 & SWS02 \\
84701470 & L 1448 S1 & S1 & 032540 & +304324 & SWS02 \\
84701471 & L 1448 S & S2 & 032541 & +304244 & SWS02 \\
\hline & & & & & &
\end{tabular}

\subsection{SWS data}

The ISO SWS instrument covered the wavelength range from 2.38 to $45.2 \mu \mathrm{m}$. The two grating spectrometers had a spectral resolution of $R \approx 1000-2000$ (AOT SWS 01,02,06; see Table 1), corresponding to a velocity resolution of $\Delta \nu \approx 150-300 \mathrm{~km} \mathrm{~s}^{-1}$. Higher resolution $(R \approx 30000)$ was achieved by inserting one of the two Fabry-Pérot filters (wavelength range 15-26 $\mu \mathrm{m}$, and 26-35 $\mu \mathrm{m}$ respectively; AOT SWS 07). The aperture of the SWS instrument is rectangular, with a size depending on the detector (i.e., wavelength). The typical aperture sizes of $14^{\prime \prime} \times 27^{\prime \prime}$ are shown in Figs. 1 and 2. For more details on the instrument and AOTs see the ISO Handbook, Volume VI: SWS - The Short Wavelength Spectrometer ${ }^{1}$ and de Graauw et al. 1996.

The data were reduced using the standard pipeline version 8.4. We deglitched and flux calibrated the spectra with the ISO Spectral Analyses Package (ISAP 1.6a). Line fluxes (Gaussian fit centred on the line) were measured above the continuum (fit by a second order polynomial). We measured the line fluxes for each observation (TDT number) separately. Then the fluxes of the same line were averaged for each of our positions (see Table 1). "Lines"

\footnotetext{
${ }^{1}$ http://www.iso.vilspa.esa.es/manuals/HANDBOOK/VI/ sws_hb/
}

with a measured FWHM significantly smaller than the instrumental profile are not included, because we do not consider them as real.

\subsection{LWS data}

With the ISO LWS instrument, observations were carried out in the wavelength range from $43 \mu \mathrm{m}$ up to $196.9 \mu \mathrm{m}$. Like with SWS, grating (AOT LWS 01,02) or FabryPérot (AOT LWS 04) modes are available. Grating scans have a resolving power between 150-300 and the spectral resolution of the Fabry-Pérot mode varies between 6800 and 9700 . The spatial resolution of the instrument is of the order of $80^{\prime \prime}$ (Swinyard et al. 1996). See the ISO Handbook, Volume IV: LWS-The Long Wavelength Spectrometer ${ }^{2}$ and Clegg et al. (1996) for details.

The data were reduced using the standard pipeline 7 . Data reduction was done similar to the SWS data, using the ISAP and LIA (version 7.3) software. Glitches due to cosmic rays were rejected and for every observation (TDT number) the line fluxes were measured. As for the SWS data, line fluxes of the same lines of spectra for each of our positions (see Table1) were averaged, again also excluding lines with $F W H M$ significantly smaller than the instrumental profile. The FP data reduction was done by standard processing and we used associated LWS 01 grating scans for optimizing the dark current. Afterwards the continuum was aligned horizontally in an interactive mode.

\section{Results}

\subsection{Cepheus $A$}

In our spectra of Cepheus A East and West, a large variety of $\mathrm{H}_{2}$ and $\mathrm{CO}$ transitions were detected. The $\mathrm{CO} 23-$ 22 line is merged with the $\mathrm{H}_{2} \mathrm{O}$ line at $113 \mu \mathrm{m}$, so only an upper limit to the individual line fluxes can be inferred. Since we have not detected the usually stronger water lines at $174.6 \mu \mathrm{m}$ and $179.5 \mu \mathrm{m}$, we attribute the flux to the CO line as an upper limit. In Cep A East we only detect the higher $(J>20) \mathrm{CO}$ lines with even $J$, because the detections are Fabry-Pérot measurements and the lines with odd $J$ were not observed with FP. For the odd $J$ lines no useful upper limits could be deduced from the full grating scans, which have a much lower S/N. Also, very strong forbidden lines of $[\mathrm{OI}]$ and $[\mathrm{CII}]$ were found in both positions. The complete list of all our detected lines in Cepheus A is given in Table 2. Due to the broad instrumental profile all the lines measured in grating mode are not resolved. CO transitions measured in Cep A East in the Fabry-Pérot mode are however resolved. Line profiles of eight lines from $\mathrm{CO}(J=14-13)$ to $\mathrm{CO}(J=28-27)$ are displayed in Fig. 3. The shifts of the lines relative to the molecular cloud at $v_{\mathrm{LSR}}=-11.15 \mathrm{~km} \mathrm{~s}^{-1}$ (Gómez et al. 1999) are $-18 \mathrm{~km} \mathrm{~s}^{-1}$. A velocity difference of $50 \mathrm{~km} \mathrm{~s}^{-1}$

\footnotetext{
${ }^{2}$ http://www.iso.vilspa.esa.es/manuals/HANDBOOK/IV/ lws_hb/
} 

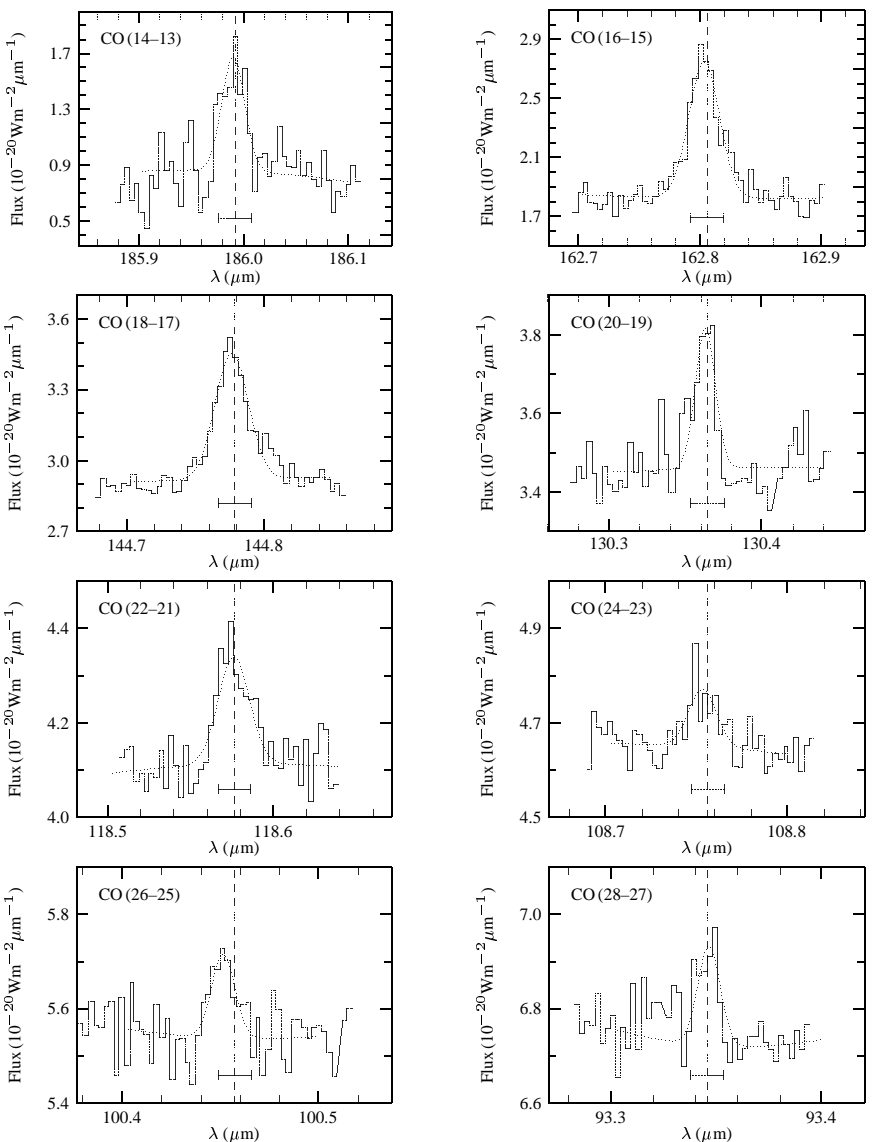

Fig. 3. Fabry-Pérot scans of CO lines in Cepheus A East. The vertical dashed lines represent the position of the line at $v_{\text {LSR }}=-11.15 \mathrm{~km} \mathrm{~s}^{-1}$ (Gómez et al. 1999). The horizontal bars indicate a velocity difference of $50 \mathrm{~km} \mathrm{~s}^{-1}$. The best fit to each line (Gaussian line + second order polynomial baseline) is given by the dotted line.

is indicated by the horizontal bar in each box. The $F W H M$ of the resolved CO lines is $49 \mathrm{~km} \mathrm{~s}^{-1}$. The resolution is insufficient to attempt to deconvolve the lines. Measurements by Vastel et al. (2000) give an instrumental profile of the LWS instrument of about $40 \mathrm{~km} \mathrm{~s}^{-1}$. This implies lines of intrinsic width not much greater than $30 \mathrm{~km} \mathrm{~s}^{-1}$. The FP measurements of CO in Cep A West did not show any lines, nor did the FP SWS observations.

\section{2. $L 1448$}

Many CO lines were detected at several positions in $\mathrm{L}$ 1448. In the outflow lobes, transitions of $\mathrm{H}_{2}$ and $\mathrm{o}-\mathrm{H}_{2} \mathrm{O}$ are also detected. As in Cep A, the $\mathrm{CO}$ and $\mathrm{H}_{2} \mathrm{O}$ lines at $113 \mu \mathrm{m}$ and also the $\mathrm{CO}$ and $\mathrm{H}_{2} \mathrm{O}$ lines at $108 \mu \mathrm{m}$ are merged. Also strong [OI] (C, N1 and N2) and [CII] (S1, $\mathrm{N} 1$ and N2) lines are found in some positions. The complete list of all measured line fluxes is given in Table 3. No velocity information is available in any of the positions in L 1448 because only grating observations were performed.
Table 2. Observed lines in Cepheus A East and West. For lines observed with SWS we only used the grating measurements, while for the fluxes from the LWS instrument mean values from both, grating and FP measurements are given.

\begin{tabular}{|c|c|c|c|c|}
\hline Element & Transition & $\lambda_{0}[\mu \mathrm{m}]$ & Cep A East* & Cep A West* \\
\hline $\mathrm{H}_{2}$ & $2-1 \mathrm{Q}(1)$ & 2.551 & & $1.4 \pm 0.6$ \\
\hline $\mathrm{H}_{2}$ & $1-0 \mathrm{Q}(9)$ & 2.560 & & $2.8 \pm 0.7$ \\
\hline $\mathrm{H}_{2}$ & $2-1 \mathrm{Q}(5)$ & 2.604 & & $1.6 \pm 0.4$ \\
\hline $\mathrm{H}_{2}$ & $1-0 \mathrm{O}(2)$ & 2.627 & $1.1 \pm 0.6$ & $3.9 \pm 0.4$ \\
\hline $\mathrm{H}_{2}$ & $1-0 \mathrm{O}(3)$ & 2.803 & & $12.2 \pm 0.4$ \\
\hline $\mathrm{H}_{2}$ & $1-0 \mathrm{O}(4)$ & 3.004 & & $3.6 \pm 0.3$ \\
\hline $\mathrm{H}_{2}$ & $1-0 \mathrm{O}(5)$ & 3.235 & & $7.1 \pm 0.3$ \\
\hline $\mathrm{H}_{2}$ & $1-0 \mathrm{O}(6)$ & 3.501 & & $1.9 \pm 0.4$ \\
\hline $\mathrm{H}_{2}$ & $1-0 \mathrm{O}(7)$ & 3.808 & & $2.4 \pm 0.6$ \\
\hline $\mathrm{H}_{2}$ & $0-0 \mathrm{~S}(12)$ & 3.995 & & $1.2 \pm 0.4$ \\
\hline $\mathrm{H}_{2}$ & $0-0 \mathrm{~S}(11)$ & 4.181 & & $5.3 \pm 1.3$ \\
\hline $\mathrm{H}_{2}$ & $0-0 \mathrm{~S}(9)$ & 4.695 & $15.1 \pm 4$ & $16.7 \pm 2.5$ \\
\hline $\mathrm{H}_{2}$ & $0-0 \mathrm{~S}(8)$ & 5.053 & $5.8 \pm 2$ & $6.2 \pm 1.8$ \\
\hline $\mathrm{H}_{2}$ & $0-0 \mathrm{~S}(7)$ & 5.511 & $19.6 \pm 4$ & $34.7 \pm 3$ \\
\hline $\mathrm{H}_{2}$ & $0-0 \mathrm{~S}(6)$ & 6.108 & $5.8 \pm 2$ & $22.4 \pm 9$ \\
\hline $\mathrm{H}_{2}$ & $0-0 \mathrm{~S}(5)$ & 6.910 & $24.9 \pm 2$ & $59.3 \pm 6$ \\
\hline $\mathrm{H}_{2}$ & $0-0 \mathrm{~S}(4)$ & 8.025 & $13.8 \pm 1.3$ & $25.7 \pm 0.5$ \\
\hline $\mathrm{H}_{2}$ & $0-0 \mathrm{~S}(3)$ & 9.665 & $9.2 \pm 1$ & $40.8 \pm 0.9$ \\
\hline $\mathrm{H}_{2}$ & $0-0 \mathrm{~S}(2)$ & 12.279 & $15.3 \pm 4$ & $31.8 \pm 1.7$ \\
\hline$[\mathrm{NeII}]$ & ${ }^{2} \mathrm{P}_{1 / 2}{ }^{-2} \mathrm{P}_{3 / 2}$ & 12.815 & & $21.8 \pm 0.9$ \\
\hline $\mathrm{H}_{2}$ & $0-0 \mathrm{~S}(1)$ & 17.035 & $7.6 \pm 0.8$ & $13.2 \pm 0.3$ \\
\hline$[\mathrm{FeII}]$ & $a^{4} F_{7 / 2}-a^{4} F_{9 / 2}$ & 17.936 & $28.3 \pm 6$ & \\
\hline$[\mathrm{SI}]$ & ${ }^{3} \mathrm{P}_{1}-{ }^{3} \mathrm{P}_{2}$ & 25.249 & $8.5 \pm 2$ & $4.9 \pm 0.5$ \\
\hline$[\mathrm{FeII}]$ & $a^{6} D_{7 / 2}-a^{6} D_{9 / 2}$ & 25.988 & $26.8 \pm 2.6$ & \\
\hline $\mathrm{H}_{2}$ & $0-0 \mathrm{~S}(0)$ & 28.219 & & $4.0 \pm 1.3$ \\
\hline$[\mathrm{SiII}]$ & ${ }^{2} \mathrm{P}_{3 / 2}-{ }^{2} \mathrm{P}_{1 / 2}$ & 34.815 & $54.7 \pm 6$ & $70.0 \pm 3$ \\
\hline$[\mathrm{FeII}]$ & $a^{6} D_{5 / 2}-a^{6} D_{7 / 2}$ & 35.349 & $15.7 \pm 5$ & \\
\hline$[\mathrm{OI}]$ & ${ }^{3} \mathrm{P}_{1}-{ }^{3} \mathrm{P}_{2}$ & 63.184 & $2150 \pm 190$ & $1360 \pm 50$ \\
\hline $\mathrm{CO}$ & $30-29$ & 87.190 & $<30$ & \\
\hline $\mathrm{CO}$ & $28-27$ & 93.349 & $28 \pm 9$ & \\
\hline $\mathrm{CO}$ & $26-25$ & 100.461 & $26 \pm 6$ & \\
\hline $\mathrm{CO}$ & $24-23$ & 108.763 & $20 \pm 6$ & \\
\hline $\mathrm{CO}$ & $23-24$ & 113.458 & & \}$<13$ \\
\hline $\mathrm{o}-\mathrm{H}_{2} \mathrm{O}$ & $4_{14}-3_{03}$ & 113.537 & & \}$<13$ \\
\hline $\mathrm{CO}$ & $22-21$ & 118.581 & $54 \pm 11$ & $<20$ \\
\hline $\mathrm{CO}$ & $21-20$ & 124.193 & & $<20$ \\
\hline $\mathrm{CO}$ & $20-19$ & 130.369 & $58 \pm 9$ & $47.1 \pm 13$ \\
\hline $\mathrm{CO}$ & $19-18$ & 137.196 & $<50$ & \\
\hline $\mathrm{CO}$ & $18-17$ & 144.784 & $161 \pm 13$ & $<50$ \\
\hline$[\mathrm{OI}]$ & ${ }^{3} \mathrm{P}_{0}-{ }^{3} \mathrm{P}_{1}$ & 145.525 & $55 \pm 7$ & $121 \pm 7$ \\
\hline $\mathrm{CO}^{+}$ & $17-16$ & 149.760 & $55 \pm 13$ & \\
\hline $\mathrm{CO}$ & $17-16$ & 153.267 & $127 \pm 32$ & $54.4 \pm 11$ \\
\hline$[\mathrm{CII}]$ & ${ }^{2} \mathrm{P}_{3 / 2}-{ }^{2} \mathrm{P}_{1 / 2}$ & 157.741 & $276 \pm 23$ & $442 \pm 20$ \\
\hline $\mathrm{CO}^{+}$ & $16-15$ & 159.100 & $123 \pm 10$ & \\
\hline $\mathrm{CO}$ & $16-15$ & 162.812 & $237 \pm 21$ & $42.5 \pm 11$ \\
\hline $\mathrm{CO}^{+}$ & $15-14$ & 169.670 & $80 \pm 9$ & \\
\hline $\mathrm{CO}$ & $15-14$ & 173.631 & $297 \pm 11$ & $81.7 \pm 18$ \\
\hline $\mathrm{CO}$ & $14-13$ & 185.999 & $300 \pm 32$ & $42.4 \pm 12$ \\
\hline
\end{tabular}

* Fluxes in Cep A East and Cep A West are in $10^{-16} \mathrm{~W} \mathrm{~m}^{-2}$.

For line profiles of the $\mathrm{H}_{2}$ lines see Fig. 3 of Nisini et al. (2000) or Fig. 5 of Nisini et al. (1999).

\section{Background to models}

\subsection{Boost factor and column density ratios}

The LWS and SWS data sets contain lines from different molecules. In the LWS data, we detect the cool CO gas, rotationally excited to the high- $J$ levels $J>14$. In the SWS data, we detect several $\mathrm{H}_{2}$ emission line fluxes (see Tables 2 and 3). 
A major obstacle in the analysis is that the LWS beam was much larger than the SWS aperture. The LWS data had a circular beam with an area of $5027 \operatorname{arcsec}^{2}$ whereas the SWS aperture is rectangular and $280 \operatorname{arcsec}^{2}$ in size in the wavelength range from $2 \mu \mathrm{m}$ to $12 \mu \mathrm{m}$. We expect, however, that the spatial extent of the high-J CO and and the rotational $\mathrm{H}_{2}$ to be comparable, both arising from cool gas. Therefore, if the cool gas extends outside the SWS aperture, we expect the measured fluxes of the CO lines to be boosted. Hence, we shall plot the observed fluxes, but apply a boost factor to the model CO fluxes to facilitate a comparison. From the $K$-band images presented here for the $1-0 \mathrm{~S}(1) \mathrm{H}_{2}$ line, we estimate a boost factor of $B(\mathrm{CO})=3-10$. Note that the model boost factors encapsulate our ignorance not only due to the ratio of the apertures but also due to the CO abundance. We shall demonstrate that models exist for which plausible boost factors are indeed predicted and abnormally high or low $\mathrm{CO}$ abundances are unnecessary.

The fluxes of detectable $\mathrm{H}_{2}$ lines typically span an order of magnitude. The derived column densities in the upper levels of the responsible transitions, however, span a range of up to five orders of magnitude even just for the ISO data, whereas errors on individual points are only $20 \%$. Therefore, to display and fit the models, it is absolutely indispensible to normalise the column densities. This is traditionally done by dividing by the columns from a $2000 \mathrm{~K}$ slab of a gas in which the level populations are in thermodynamic equilibrium (e.g. Brand et al. 1988). Although, for the ISO data, a $1000 \mathrm{~K}$ slab would be more appropriate, we remain with the $2000 \mathrm{~K}$ normalisation in order to avoid confusion. Hence, in the Column Density Ratio diagrams, we actually display for each line the quantity

$\log \left[\frac{C D R}{C D R(2000 \mathrm{~K})}\right]=\log \frac{N}{N_{\mathrm{o}}}-\log \frac{N_{2000}}{N_{\mathrm{o}}}$

where $N$ is the observed column in the upper energy level, $N_{2000}$ is the column of an arbitrary slab at $2000 \mathrm{~K}$, and $N_{\mathrm{o}}$ is a constant chosen so that the column derived from a specific line is unity. For ground-based observations, usually the upper level of the 1-0 S(1) line is used. Here, we employ the $0-0 \mathrm{~S}(5)$ line.

\subsection{Model range}

Models predictions relevant to near-infrared observations of warm molecular gas (i.e. $1500 \mathrm{~K}-4000 \mathrm{~K}$ ) are discussed by Eislöffel et al. (2000). Here, we classify the models in the context of cool molecular gas (i.e. $300 \mathrm{~K}-1500 \mathrm{~K}$ ).

Model 1TEMP. A reservoir of constant temperature gas, assuming LTE, produces a straight line on the $\log (\mathrm{CDR})$ diagram. The gradient yields the excitation temperature. The model is unrealistic for the cool gas since the cooling time at $\sim 300-1500 \mathrm{~K}$ is less than a year. There is no mechanism to maintain a specific clump of gas at these elevated temperatures. The rotational $\mathrm{CO}$ emis- sion diagrams for 1TEMP were published by McKee et al. (1982).

Model NTEMP. More than one co-existing constant temperature component is equally implausible since there are no equilibrium temperatures or multi-phase media expected in the temperature range in which $\mathrm{H}_{2}$ is detected. Two component fits can appear successful when column densities are plotted (e.g. Everett et al. 1995 for OMC-1), but clearly fail the more sensitive CDR analysis (Burton \& Haas 1997).

Model CSHOCK. A planar C-type shock, in which ion-neutral friction heats the gas in a thick shock layer (Draine et al. 1983). The predicted CDRs are similar to 1TEMP but with slight curvature at low $T_{j}$ (see Fig. 3 of Smith et al. 2000). The excitation temperature of a $C$-shock is sensitive to the density, ion fraction, magnetic field strength, magnetic field direction, shock speed and oxygen chemistry (Smith \& Brand 1990). These parameters cannot be uniquely extracted through modelling. The CO predictions were presented by Smith (1991). A maximum CO flux occurs at some $J$-value, depending on the density and maximum temperature.

Models $C T / C T F$ Time-dependent $C$-shocks. $C$-shocks with moderate to high Alfvén numbers ( $>5$ for a transverse field) are unstable (Model CTU) (Wardle 1990). Instability alters the resulting CDR behaviour by increasing the quantities of hot and cold gas, steadily increasing the CDR curvature (Fig. 16 of Mac Low \& Smith 1997). $C$-shocks may also require excessive formation times (Model CTF). The time to reach the steady state CDR (Fig. 12 of Smith \& Mac Low 1997) beginning from a jump shock structure is about one flow time.

Model $C B O W$. A supersonic flow past an obstacle creates a curved shock surface. Each part of the surface will excite the molecules according to the local conditions. This yields convex CDR curves in which the excitation increases with upper energy level. For a $C$-type shock surface, the curve is independent of the bow speed provided the speed exceeds the molecular breakdown speed (i.e. the bow apex corresponds to a hot dissociated cap and the $\mathrm{H}_{2}$ emission is distributed in fixed warm, cool and cold components in the wings). The CDR curves still depend on the bow geometry, departure from LTE, and the oxygen abundance (Smith et al. 1991a, 1991b). Highly aerodynamic bows have been suggested by the overall lower excitation in Cepheus E (Eislöffel et al. 1996). The CO diagram does not display a maximum in the high- $J$ range (due to the cool molecular component) (Smith 1991).

Model CABSORBER. In the shock absorber model, a $C$-type bow with a high upstream Alfvén speed generates wide $\mathrm{H}_{2}$ profiles (Smith et al. 1991b). This model is able to accelerate molecules to high speed without dissociating them. Relatively less cool gas results in a maximum in the CO diagram at high- $J$ (Smith 1991).

Model TURB. Supersonic turbulence is generated by a jet, wind or in the wake of a curved shock. The turbulence leads to shocks which then dissipate, interact and disperse. The shock spectrum is an exponential function 
Table 3. Observed lines in L 1448.

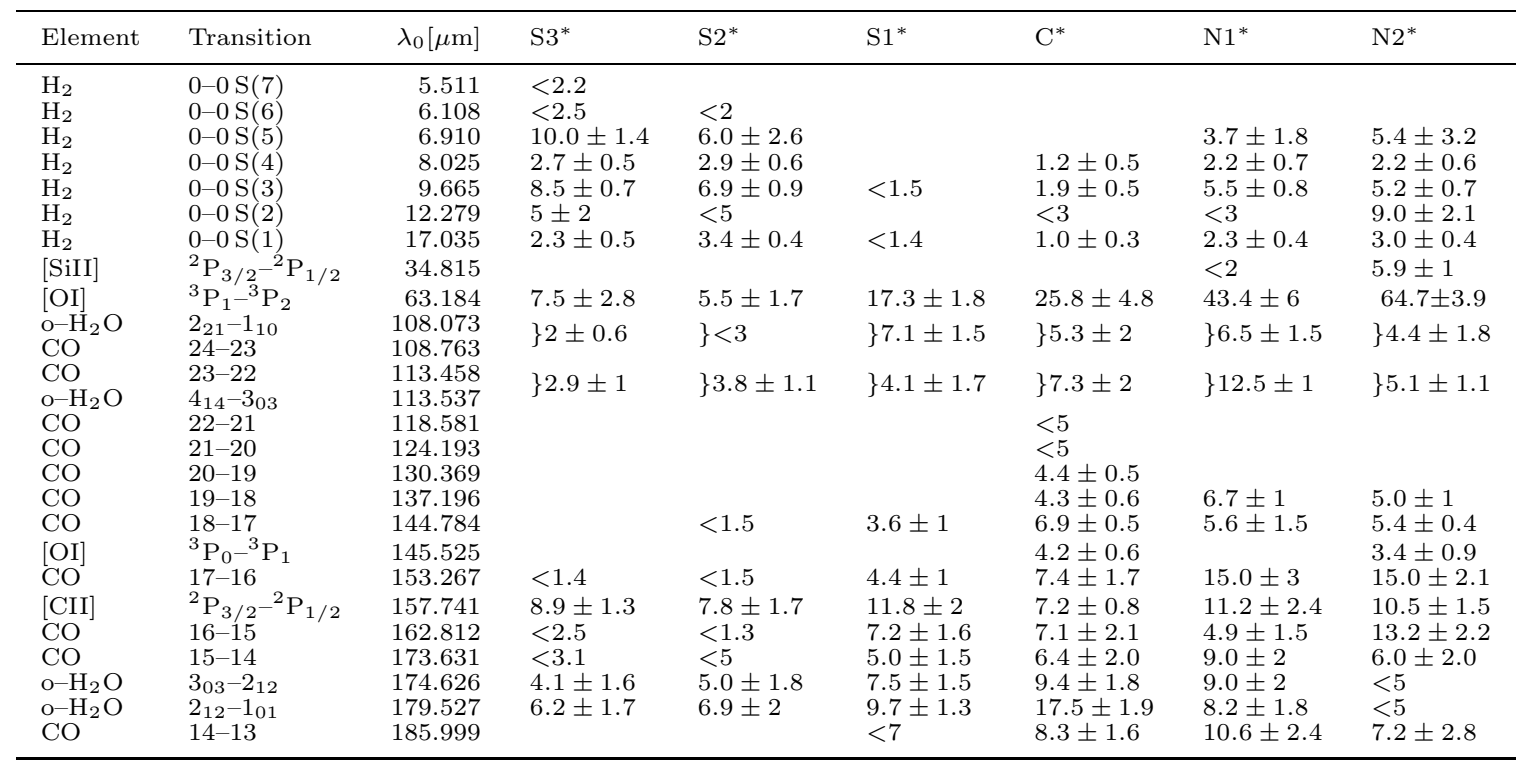

* Fluxes in $\mathrm{L} 1448$ are in $10^{-16} \mathrm{~W} \mathrm{~m}^{-2}$.

of velocity and time which suggests that weak shocks dominate the molecular excitation properties (Smith et al. 2000). Both $J$-type, TURBJ, and $C$-type, TURBC, versions would predict low excitation (Smith et al. 2000).

Model STR. A quasi-steady velocity cascade, termed a "Supersonic Turbulent Reactor" dissipates energy in shocks at the same rate as it is injected. Uniform driven turbulence generates a power-law spectrum of shocks following an inverse-square root law with the shock jump speed. The molecular excitation, still to be calculated, would probably be high.

Model JSLOW. Non-dissociative planar radiative shocks generate high excitation CDR curves $(3000-3600 \mathrm{~K})$. Lower excitations are only possible with a high magnetic field or a carefully chosen shock velocity (Smith 1994a). The JSLOW model requires reasonably high ion/neutral fraction $\left(\chi>10^{-5}\right)$. A CO diagram will be presented here.

Model JFAST. A dissociative front is followed by cooling and molecule reformation at $T \sim 500 \mathrm{~K}$. No detailed CDRs are available. However, this model is characterised by high $2-1 \mathrm{~S}(1) / 1-0 \mathrm{~S}(1)$ ratio, low intensities and cascade signatures (i.e. strong fluoresence lines, Hollenbach \& McKee 1989). Also immediately distinguished by the strong atomic hydrogen emission (including the infrared lines $\mathrm{Br} \gamma, \mathrm{P} \beta$ etc.). $\mathrm{CO}$ diagrams show maxima typically at $J \sim 15-25$ (Hollenbach \& McKee 1989) for densities above $10^{4} \mathrm{~cm}^{-3}$.

Model JBOW. A curved $J$-type shock in which molecular emission is dominated by extended wings of Model JSLOW type (Smith 1994a). To reduce the excitation to observed levels often requires extremely long weak shock sections.
Model FLO. X-ray and UV radiation heats and excites the molecules by UV pumping, via electron collisions and by direct dissociation and consequent reformation. A fluorescent spectrum is produced. Each vibrational level produces a distinct CDR curve (e.g. McCartney et al. 1999, Black \& van Dishoeck 1987). High- $J$ CO emission lines are not expected from photodissociation regions since the $\mathrm{CO}$ layer lies deeper into the cloud where the temperatures are low. Mid- $J$ CO can, however, be very strong, as observed, if the PDR is highly clumped.

Model FLY. UV radiation from nearby shocks excites the molecules by Lyman resonance excitations (Black \& van Dishoeck 1987). Model FLY may be relevant to bow shocks, with the Ly $\alpha$ emission generated in strong shocks across the bow cap (Fernandes \& Brand 1995).

Model PREC. Magnetic precursors may lead slow planar $J$-type shock provided the ion fraction is low. Precursor development, found and modelled within Model CTU, produces quasi-linear CDRs (Smith \& Mac Low 1997). This is the "neutral transformation" stage (Stage 3) which can last for a time of order of 100-1000 years. In fast shocks, pre-shock ionisation inhibits a magnetic precursor.

\section{Interpretation: Cepheus A east}

\subsection{Intrinsic and extrinsic factors}

Consistent interpretations of the ISO data have proven evasive. Nisini et al. (1999), for example, derived a $\mathrm{CO} / \mathrm{H}_{2}$ abundance ratio of $\sim 0.01$ in $\mathrm{L} 1448$, two orders of magnitude higher than in quiescent molecular gas. One may then invoke solutions such as the gas being 99\% atomic (Nisini et al. 1999). This, however, creates a worse problem since $\mathrm{H}_{2}$ would reform on grains in a very short time 
$(\sim 30 \mathrm{yr})$ at the high atomic densities required. Instead, we show here that many such problems do not arise if we employ models which involve a distribution in shock strengths.

First, we discuss the importance of extinction and radiation. Dust extinction alters the rotational $\mathrm{H}_{2}$ O-0 emission lines systematically, yielding an apparent gas excitation lower than the intrinsic value. The extinction can e.g. be estimated from the $0-0 \mathrm{~S}(3)$ line. This line at $9.7 \mu \mathrm{m}$ lies in the middle of a silicate absorption feature which reduces the line flux by a factor which we can estimate from the flux relative to the neighbouring $\mathrm{S}(2)$ and $\mathrm{S}(4)$ column densities. On assuming the extinction is from within molecular gas, the optical depth $\tau$ at $9.7 \mu \mathrm{m}$ translates into a $K$-band extinction of $\mathrm{A}_{k}=0.85 \tau$ (Whittet 1988). We thus estimate a $K$-band extinction of 1.2 magnitudes, as shown in the figures below. (The 0-0 S(3) line may also display a depressed flux because the ortho-para ratio is lower than 3 . We find that this may alter the derived densities by a factor of $\sim 2$.) A standard differential extinction law in which relative fluxes are increased by $0.4 \mathrm{~A}_{k}\left(\left(\lambda_{k} / \lambda\right)^{1.7}-1\right)$ was used.

We deduce that the first vibrational level is radiatively depopulated rather than in LTE. This constrains the molecular and atomic densities. This result is supported by the observations of Cep A West for which the non-LTE effect is well established since several higher vibrational lines need to be modelled (see Sect.6).

There are several strands of evidence which support shock excitation rather than photon excitation models FLO and FLY. Firstly, the CO line profiles in Cepheus A East are resolved (Fig. 3). This implies that supersonic flow at speeds capable of providing collisional excitation is present. Secondly, the columns of gas in levels from ortho and para $\mathrm{H}_{2}$ are consistent with an ortho-para ratio of 3 , suggesting modification to the value of 3 within warm gas, consistent with shocks (see below). Fluorescence mechanisms would not be expected to alter this ratio. Thirdly, the $\mathrm{H}_{2}$ line ratios are consistent with thermal excitation whereas photon dissociation regions display strongly nonthermal population distributions. Thus, we shall not entertain further the photon excitation mechanisms.

We take as fiducial parameters the abundances $\epsilon(\mathrm{CO})=2 \times 10^{-4}, \epsilon(\mathrm{C})=10^{-4}, \epsilon(\mathrm{He})=10^{-1}$ and $\epsilon(\mathrm{O})=4 \times 10^{-4}$, although we shall find that $C$-type bows require much lower atomic oxygen levels. We take a uniform magnetic field with an Alfvén speed $v_{\mathrm{A}}=2 \mathrm{~km} \mathrm{~s}^{-1}$ in the pre-shocked medium. An ion abundance of $2 \times 10^{-7}$ is assumed for the $C$-type shocks with the ions conserved during the shock passage.

\subsection{JSLOW}

Although shock surfaces are most likely curved or distorted, we attempt to fit the data with non-dissociative planar $J$-shocks of speed $10-22 \mathrm{~km} \mathrm{~s}^{-1}$. In Fig. 4, we employ the relatively low $1-0 \mathrm{O}(2)$ column density to deter-
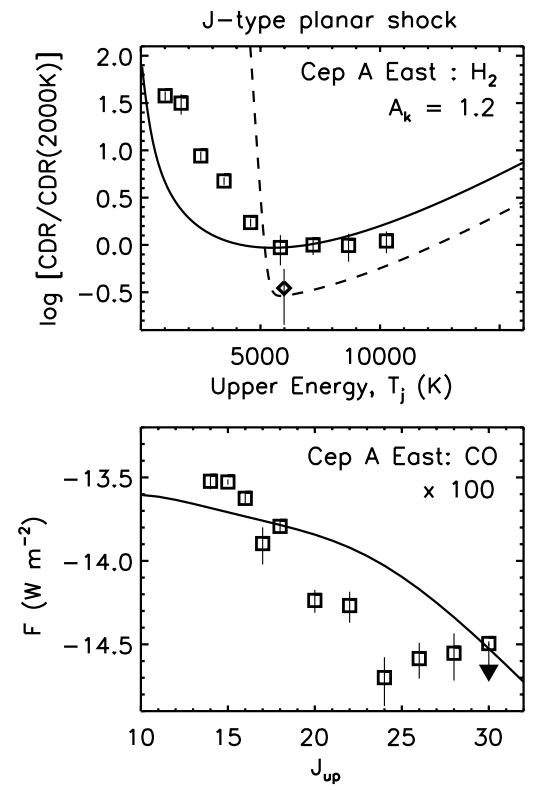

Fig. 4. Model JSLOW: a $J$-shock with speed $15 \mathrm{~km} \mathrm{~s}^{-1}$ and pre-shock density of $2 \times 10^{4} \mathrm{~cm}^{-3}$ is displayed against the $\mathrm{H}_{2}$ (top) and CO (bottom) data for Cep A East. Shown are the ground vibrational level (full line) and the first excited level of $\mathrm{H}_{2}$ (dashed line) which is radiatively depopulated and fitted to the $1-0 \mathrm{O}(2)$ line (diamond symbol). Abundances are $\epsilon(\mathrm{O})=$ $4 \times 10^{-4}$ and $\epsilon(\mathrm{CO})=2 \times 10^{-4}$ and $\epsilon(\mathrm{C})=10^{-4}$.

mine the pre-shock density (assuming the upstream gas is molecular). We have fully explored the parameter space in our modelling. On assuming a higher density, the CO would become stronger relative to the $\mathrm{H}_{2}$. A lower density, combined with an increased atomic fraction to maintain the population of the first vibrational level, generates models with less CDR curvature than required. Finally, the surface brightness of the shock must also be consistent with the observed line strengths, as discussed below. The CO rotational emission line fluxes generated in the JSLOW models however are a factor of 100 too low on the assumption that all the $\mathrm{CO}$ and $\mathrm{H}_{2}$ is from within the smaller SWS aperture (lower panel of Fig. 4).

We remark that the JSLOW model does not reproduce the $\mathrm{CO}$ rotational excitation either. It has been argued that a lower excitation spectrum would be produced in the JSLOW model if the water abundance is kept low, so that the cooler gas persists longer (Brand et al. 1988). Smith (1994b), however, showed that this would require water abundances well below $10^{-7}$ to explain the excitation typically found in protostellar outflows. At this level, both $\mathrm{CO}$ and gas-grain cooling have taken over as fast coolants of the cool gas, maintaining the high excitation of the model.

Very slow planar $J$-shocks, with speeds in the range $8-10 \mathrm{kms}^{-1}$, or very high Alfvén speeds, with $v_{\mathrm{A}}=$ $6-7 \mathrm{~km} \mathrm{~s}^{-1}$, also produce low excitation $\mathrm{H}_{2}$ spectra (Smith 1994b). It is implausible, however, that such a narrow parameter range would occur over such a large area. 

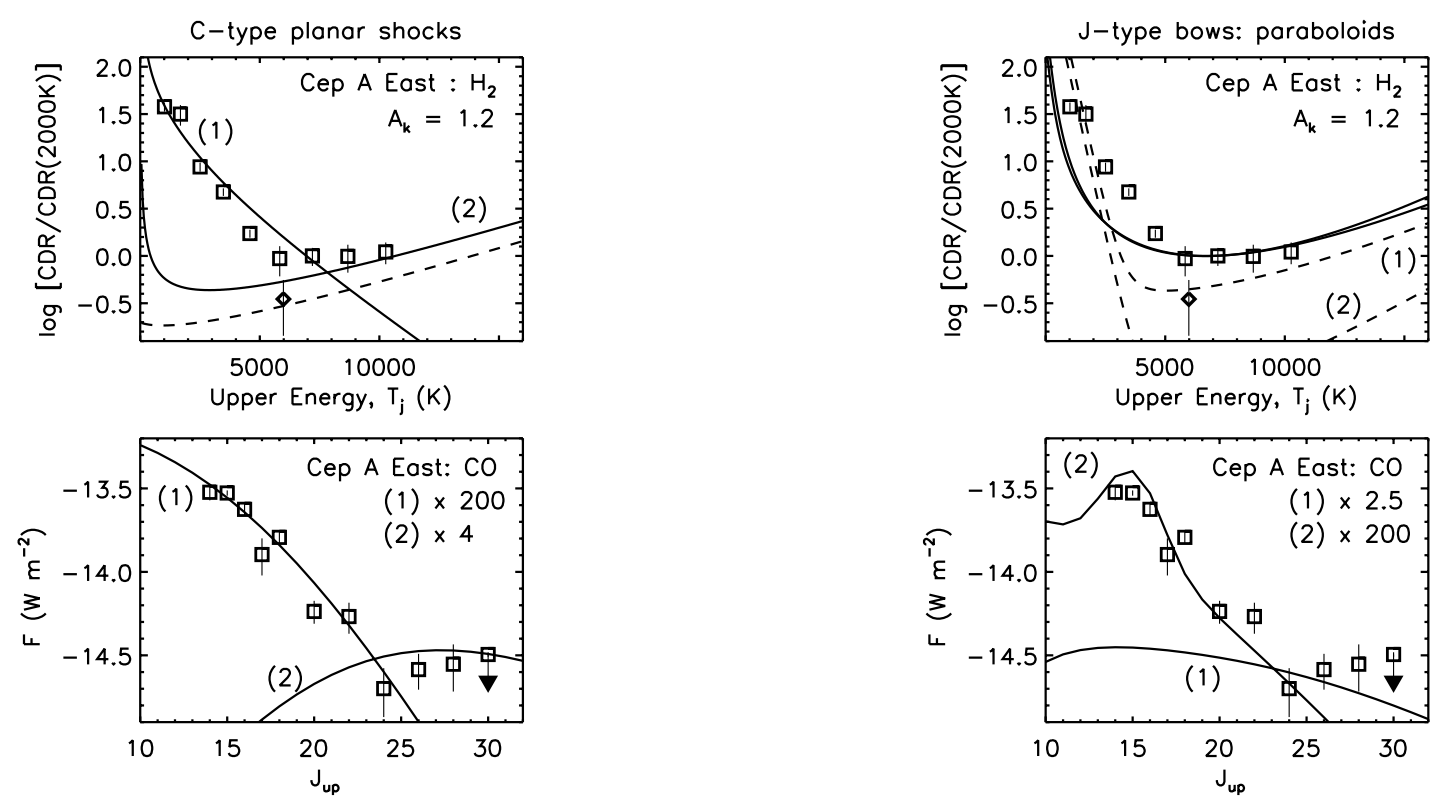

Fig. 5. Model CSHOCK: two $C$-shocks with maximum temperatues of (1) $1164 \mathrm{~K}$ and (2) $3367 \mathrm{~K}$ are displayed against the $\mathrm{H}_{2}$ (top) and $\mathrm{CO}$ (bottom) data (see text). The full lines are the ground vibrational level, and the dashed is the first excited level which is radiatively depopulated. At the low temperature and density of the cool shock, the upper level is vacant.

This leads us to abandon the JSLOW model, as also concluded for many Herbig-Haro objects (Smith 1994b).

\subsection{CSHOCK}

We show here that planar $C$-shock models require a contrived dynamical situation to be consistent with the data. The $C$-shock models which fit the data are shown superimposed on the Cep A East data in Fig. 5. Note, however, that $C$-shock excitation varies strongly with shock speed and other parameters.

A single $C$-shock generates a near $\operatorname{linear} \log (C D R)-T_{j}$ relation. Hence two $C$-shocks can fit the Cep A East $\mathrm{H}_{2}$ data quite well. These two-shock fits determine the maximum temperatures of the shocks, which can be interpreted as shock velocity and/or ionisation fraction differences. To simultaneously model the $\mathrm{CO}$ line excitation requires a low density for the low-excitation shock and a high density for the hot shock. The densities and velocities of the two components displayed are $2 \times 10^{6} \mathrm{~cm}^{-3}$ and $30 \mathrm{~km} \mathrm{~s}^{-1}$ (hot) and $3 \times 10^{4} \mathrm{~cm}^{-3}$ and $25 \mathrm{~km} \mathrm{~s}^{-1}$ (cold).

The CSHOCK model is unable to explain the CO flux levels. To model the relative level of $\mathrm{CO}$ emission, assuming a $\mathrm{CO}$ abundance of $2 \times 10^{-4}$, requires that the shocks seen in $\mathrm{CO}$ fill an area of 200 and 4 times more than the areas seen in the $\mathrm{H}_{2}$ observations, for the cool and warm shocks, respectively. This would implie that the warm shocks were mainly contained within the small SWS aperture whereas the cool shocks were spread throughout the larger LWS beam. However, since the LWS beam is only 18 times larger, the cool shocks must actually con-

Fig. 6. Model JBOW: paraboloidal $J$-shocks with speed $80 \mathrm{~km} \mathrm{~s}^{-1}$ and pre-shock densities of $10^{6} \mathrm{~cm}^{-3}$ and $10^{4} \mathrm{~cm}^{-3}$ are displayed against the $\mathrm{H}_{2}$ (top) and $\mathrm{CO}$ (bottom) data for Cepheus A East. The full line is the ground vibrational level, and the dashed line is the first excited level of $\mathrm{H}_{2}$. Note: the higher density model is closer to LTE and the CO excitation is higher.

spire to strongly avoid the warm SWS region. The high observed CO in Cepheus A East can thus be explained if the molecular gas in the SWS aperture is largely swept up into dense warm shocks, while cool gas and weaker shocks occupy the surroundings contained in the LWS beam. This model thus predicts a "shocked halo". It does seem improbable, however, that the halo avoids the SWS aperture so perfectly even in projection.

\subsection{TEMP and NTEMP}

From the CDR diagrams, we notice that the $\mathrm{H}_{2}$ columns are not distributed exponentially (i.e. with $\log (C D R) \propto$ $T_{j}$ ) in Cepheus A or L 1448, eliminating 1TEMP. Model NTEMP, with two components, would appear to be consistent with the Cepheus A data, with the components corresponding quite closely to the shocks derived in the CPLANAR modelling above. The model, however, runs into the same problem as found for the CSHOCK model: to fit the high- $J \mathrm{CO}$ data with the same cool gas component that fits the $\mathrm{H}_{2}$ data requires an excessively high boost factor between the emission contained in the LWS and SWS apertures.

\subsection{JBOW}

We examine bow shocks models with geometries of the form

$Z \propto R^{s}$ 

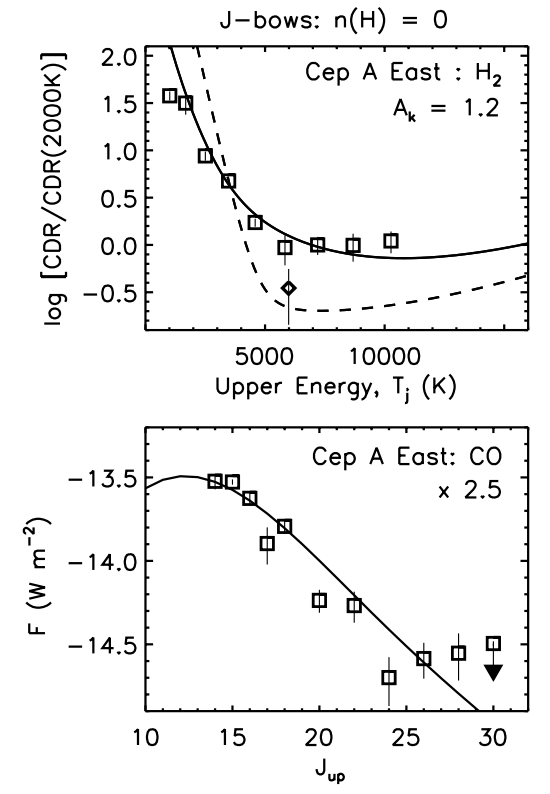

Fig. 7. Model JBOW: extremely long wings to the bow create more cool gas: the bow shape parameter is $s=1.25$. The gas is fully molecular. A pre-shock density of $10^{6} \mathrm{~cm}^{-3}$ is then required to explain the non-LTE $\mathrm{H}_{2}$ distribution, which then neccessitates a very reasonable $\mathrm{CO}$ boost factor.

in cylindrical coordinates. These are often equivalent to power law number-velocity distributions of the form $\mathrm{d} N / \mathrm{d} v \propto v^{\alpha}$, with the power law index related to $s$ by $\alpha=2 s /(s-1)$.

First, paraboloidal $J$-shocks predict high excitation $\mathrm{H}_{2}$ $K$-band spectra, and, as can be seen from Fig. 6 , not as much of the cool gas as detected by the ISO. The models are clearly also inconsistent with the $\mathrm{CO}$ data.

A bow with extended wings is thus suggested, the wings producing the extra cool $\mathrm{H}_{2}$. After much modelling, we find that $s=1.25$ is required: extremely long wings to the bow. The bow then appears as an extremely long thin edge-brightened cone for the $\mathrm{H}_{2}$ and $\mathrm{CO}$ lines observed with ISO, as shown in Fig. 12. Accepting this structure, there is a choice of models which can explain most of the data: either some fraction of atomic hydrogen is present in the upstream gas at a quite low density $(n(\mathrm{H})=0.2 \mathrm{n}$ and $n=3 \times 10^{4} \mathrm{~cm}^{-3}$, not displayed) or a pure molecular gas but with a quite high density, as shown in Fig. 7 .

The boost factor for the CO differentiates the models, with the low density model requiring a boost factor of several hundred. This is not realistic since the areas sampled by the LWS beam is only 18 times larger, and the SWS beam is centred on the brightest $\mathrm{H}_{2}$ outflow region.

On the other hand, the high density case yields neatly a consistent picture in which the larger LWS beam contains only a few times more shocked gas than the SWS beam. This difference between the models resolves the problems encountered by Nisini et al. (1999) and others, who uncovered similar high boost factors. Thus, distributions of shocks, with many weak shocks in a high density medium, generate a peak $\mathrm{CO}$ flux at low $J$ despite the high den-
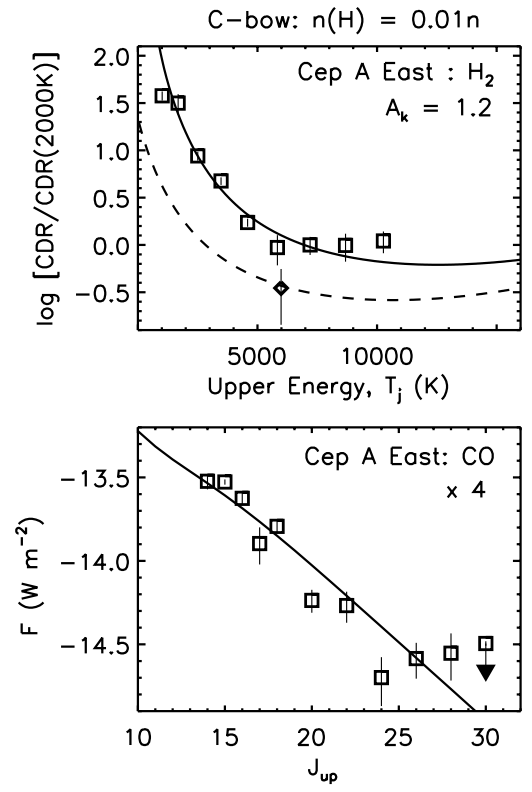

Fig. 8. Model CBOW: A $C$-type bow with speed $100 \mathrm{~km} \mathrm{~s}^{-1}$ and pre-shock density $10^{6} \mathrm{~cm}^{-3}$, ion fraction $2 \times 10^{-7}$ and bow geometry $s=1.4$. The oxygen abundance is low here: $10^{-4}$. The full line is the ground vibrational level, the dashed line the first excited level of $\mathrm{H}_{2}$.

sity. Hence it is the dynamics, which sets the distribution of shocks, which determines the overall excitation, while the actual temperatures of individual shocks play a secondary role.

\section{6. $C B O W$}

A high density $C$-type bow produces an excellent fit (Fig. 8). The uncomfortable geometric factor encountered in the $J$-bow model is also overcome: the bow geometry is $Z \propto R^{s}$ with $s=1.4$ i.e. the wings are not so extremely extended, as shown in Fig. 13. Rather than long wings, we propose that the emission from cool molecules arises in the wakes of bow shocks, within which supersonic vorticity is dissipated in further weak shocks.

The $C$-bow model predicts a reasonable $\mathrm{CO}$ boost factor of $B(\mathrm{CO})=4$. Note that one per cent of atomic hydrogen is predicted for the best fit - this reduces the density which is necessary to reduce the predicted $\mathrm{CO}$ fluxes into the range consistent with the observations. An even lower density, however, reduces the curvature of the CDR curve and so degrades the fit.

\subsection{CABSORBER}

The high magnetic field CBOW model provides an alternative interpretation (not displayed). A lower density is necessary to fit the mid- $J$ CO lines. This leads to increased, but still plausible, boost factors for Alfvén speeds up to $\sim 20 \mathrm{~km} \mathrm{~s}^{-1}$. The essential difference between the CABSORBER and CBOW models lies in the low- $J$ CO levels. The CABSORBER predicts a turnover in the 

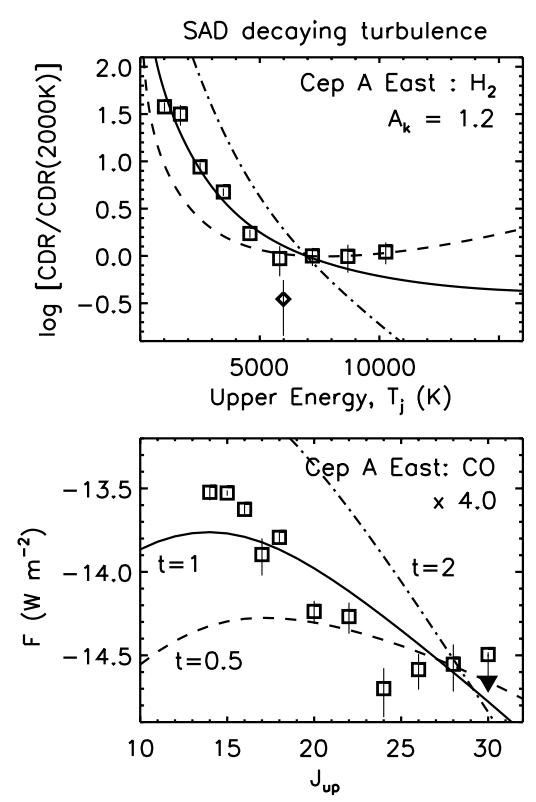

Fig. 9. Model TURBC: shock distribution during the decay of supersonic turbulence. Normalised times are $t=0.5,1.0,2.0$ (dashed, solid, dot-dashed).

fluxes at $J \sim 15$. For the particular case of Cep A East, the lower- $J$ fluxes continue to rise with decreasing $J$, consistent with the low magnetic field CBOW model.

\subsection{Turbulence: TURB and STR}

We now consider supersonic decaying turbulence, taking the distribution of shocks from 3D simulations (Smith et al. 2000), and apply the planar $C$-shock model. We thus model supersonic ambipolar diffusion (SAD) i.e. a turbulent spectrum of $C$-shocks. The excitation is shown at three times in Fig. 9, from the earliest and hottest (dashed) to the coolest (dot-dash).

From Fig. 9, it appears that Cepheus A East could well be in a state of decaying turbulence at a time corresponding to about one crossing time at the root mean square speed over the scale at which turbulent energy was injected.

The CO model flux has been boosted by a factor to fit the observed CO flux levels, corresponding to (1) the aperture size difference (i.e., only $1 / 4$ of the $\mathrm{H}_{2}$ is in the aperture) or/and (2) the $\mathrm{CO}$ abundance exceeds the chosen value of $2 \times 10^{-4}$.

\subsection{Cepheus A East: Further diagnostics}

The model values for the $\mathrm{H}_{2}$ fluxes can be adjusted to the observed value by means of a shock filling factor: the ratio of the shock front area to the observed area. A $J$-type bow shock of shape $(z / L)=(1 / s)(R / L)^{s}$ generates the $0-0 \mathrm{~S}(5)$ emission in the wings down to oblique shock angles corresponding to transverse shock speeds of $\sim 8 \mathrm{~km} \mathrm{~s}^{-1}$. At this location, the distances from the apex and the axis are denoted as $z_{0}$ and $R_{0}$. For the JBOW models displayed here with $s=1.25$, we choose a bow speed of $80 \mathrm{~km} \mathrm{~s}^{-1}$. The resulting average surface brightness will be expressed here over an area $2 z_{0} R_{0}$. This will actually be independent of the chosen velocity.

The predicted bow-averaged surface brightnesses for the JBOW displayed in Fig. 7 is then $550 \times 10^{-6} \mathrm{~W} \mathrm{~m}^{-2}$ for the $0-0 \mathrm{~S}(5)$ line. This is an extremely high flux for $J$-shocks, resulting from the low s-values: the extended shock wings are dominant infrared emitters. The observed extinction-corrected flux is $5.7 \times 10^{-6} \mathrm{~W} \mathrm{~m}^{-2}$. Hence, a low surface filling factor for the bow of 0.01 is required.

For the $C$-bow model, the predicted bow-averaged surface brightness is $21 \times 10^{-6} \mathrm{~W} \mathrm{~m}^{-2}$. The surface filling factor in the SWS aperture is then 0.4. This is highly plausible given the infrared image in the $1-0 \mathrm{~S}(1)$ line. In the CBOW model, the power emitted is scalable: one can consider a lower magnetic field or a higher fraction of ions (e.g. long-lived metal ions) which both produce the same infrared emission but from slower shocks. For example, a model with an ion fraction of $2 \times 10^{-6}$ is found to increase the surface filling factor by a factor of 6 .

We remark that with densities of order of $10^{6} \mathrm{~cm}^{-3}$, total shock powers are typically of order $10^{-3} \mathrm{~W} \mathrm{~m}^{-2}$. As we have seen, these densities are necessary to produce the high levels of $\mathrm{CO}$ rotational emission as well as producing better fits to the $\mathrm{H}_{2}$ excitation.

In bow shocks, the cool gas responsible for oxygen emission is present in the long cool tails while the oxygen is rapidly transferred into water molecules in the stronger $C$-shock section nearer to the bow apex. The [OI] $(63 \mu \mathrm{m})$ line within the LWS beam has an average surface brightness of $2.3 \times 10^{-5} \mathrm{~W} \mathrm{~m}^{-2}$ and the flux ratio of $[\mathrm{OI}](63 \mu \mathrm{m}) / \mathrm{CO}(J=20-19)=37$. We find that with an oxygen abundance of $4 \times 10^{-4}$, the intensity ratio of $[\mathrm{OI}](63 \mu \mathrm{m}) / \mathrm{CO}(J=20-19)=424$ is predicted by the CBOW model. Reducing the oxygen abundance to $10^{-4}$, however, also boosts the $\mathrm{CO}$ which is now the main coolant below $1000 \mathrm{~K}$. Then the intensity ratio of $[\mathrm{OI}](63 \mu \mathrm{m}) / \mathrm{CO}(J=20-19)=39$ and 70 for $\mathrm{CO}$ abundances of $2 \times 10^{-4}$ and of $4 \times 10^{-4}$, respectively. The latter model is the one displayed in Fig. 8. Note, however, that (1) there may well be a large contribution resulting from the far-ultraviolet excitation of this region and (2) the $63 \mu \mathrm{m}$ line is probably optically thick (e.g. Caux et al. 1999).

The JBOW models employed above, however, generate [OI] (63 $\mu \mathrm{m}$ emission typically $200-1000$ larger than the individual $\mathrm{CO}$ rotational emission lines. This is due to the large surface area of very cool shocks for which the oxygen is not converted and in which it is the dominant coolant. Hence, JBOW models can certainly be excluded in explanations of low-excitation $\mathrm{H}_{2}$ spectra.

\subsection{Cepheus $A$ East: The $\mathrm{H}_{2}$ ortho-para ratio}

The $0-0 \mathrm{~S}(1)$ and $0-0 \mathrm{~S}(3)$ fluxes both appear to be lower than predicted by the models, which assume a fixed 

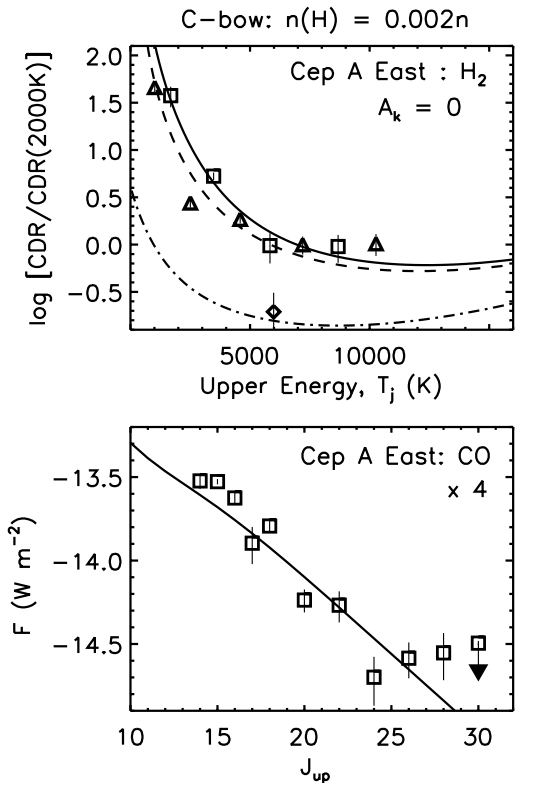

Fig. 10. Model CBOW: the ortho-para ratio is modelled here by taking an initial ratio of 1 . Just 0.2 per cent of atomic hydrogen is required here. The other parameters are as in Fig. 8. The full line and squares represent the model and data for the para state, and the dashed line and triangles for the ortho state.

ortho-para ratio of 3 , the equilibrium high-temperature value. We note that the higher $0-0$ lines show no sign of deviation due to a low ortho-para ratio (see Fig. 10). In particular, the $0-0 \mathrm{~S}(5)$ is consistent with an ortho-para ratio of 3 . This suggests that the lowest $\mathrm{H}_{2}$ rotational lines arise in distinct shocks in which the gas has never been strongly heated, rather than the re-cooled layers of strong shocks. Then, the ortho-para ratio would be modified to the equilibrium value only in the higher rotational lines. The mechanism to achieve this is reactive collisions with atomic hydrogen which indeed has an activation barrier of $3200 \mathrm{~K}$. This is evidence for a full spectrum of shock speeds. We expect this mechanism to be active in $C$-shocks (Smith et al. 1997). In $J$-shocks, however, the impulsive heating ensures immediate modification to the equilibrium ratio.

\subsection{The hot molecular component}

A distinct hot component, not predicted by the bow models, is inferred from the increase of CO line flux with $J$ above 25, although the data are poor. The existence of this component is quite plausible since it is also found in LWS observations of T Tauri, IC 1396N and L 1448-mm (Di Giorgio et al. 1999). It is notable that all the detections are associated with beams very close to the associated driving sources. A hot shock superimposed on the bow is thus suggested. A steady $C$-shock with a density exceeding $1.4 \times 10^{6} \mathrm{~cm}^{-3}$ and a temperature $\sim 4000 \mathrm{~K}$ or a planar $J$-shock (Fig. 11) provide good fits.
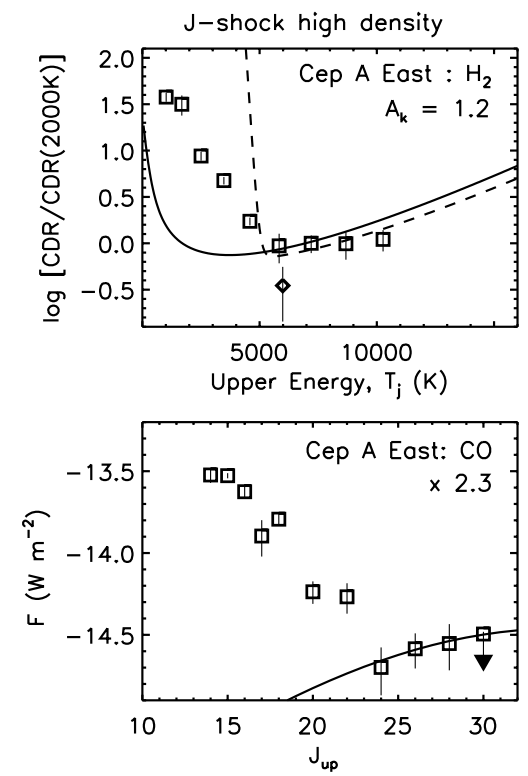

Fig. 11. Model JSLOW: a high density $J$-shock to interpret the hot $\mathrm{CO}$ gas. A shock with speed $15 \mathrm{~km} \mathrm{~s}^{-1}$ and pre-shock density of $2 \times 10^{6} \mathrm{~cm}^{-3}$ is displayed against the data for Cepheus A East.

The proximity to the driving source then suggests that a fast wide-angle wind from the driving source drives into the ambient medium at high speed. It is preceeded by an ionised precurser due to the UV emission produced in the reverse shock. The forward shock would then be $J$-type. Two-component shocks also arise during $C$-shock formation (model CTF). In this case, a $C$-type magnetic precurser precedes a weakening $J$-shock in a formation time of order of $100 \mathrm{yr}$. This model is characterised by the spatial distribution: the cool precurser leads the warm $J$-shock.

\subsection{Cepheus A East: The CO line profiles}

We display the predicted images, velocity-position diagrams and line profiles for the $\mathrm{CO} J=18-17$ transition which is the measured rotational CO line with the highest signal-to-noise ratio. At present, we can only model single bow shocks, and assume that the shock thickness is negligible relative to the bow transverse width. The LWS beam is, however, large and many such bows are probably contained within the aperture. Line broadening would then be dominated by the large scale dynamics i.e. by the superimposed profiles from numerous bow shocks moving in various directions, with different speeds into media with different velocities. Given these considerations, a bow model will be implausible if a single bow predicts lines with widths above the observed $F W H M$ of $49 \mathrm{~km} \mathrm{~s}^{-1}$ or the intrinsic $F W H M$ of $\sim 30 \mathrm{~km} \mathrm{~s}^{-1}$.

The model data shown in Figs. 12 and 13 illustrate that very narrow $\mathrm{CO}$ lines are indeed predicted for single bow shocks of both $J$ - and $C$-type for all conditions which could explain the low molecular excitation. The lines are 

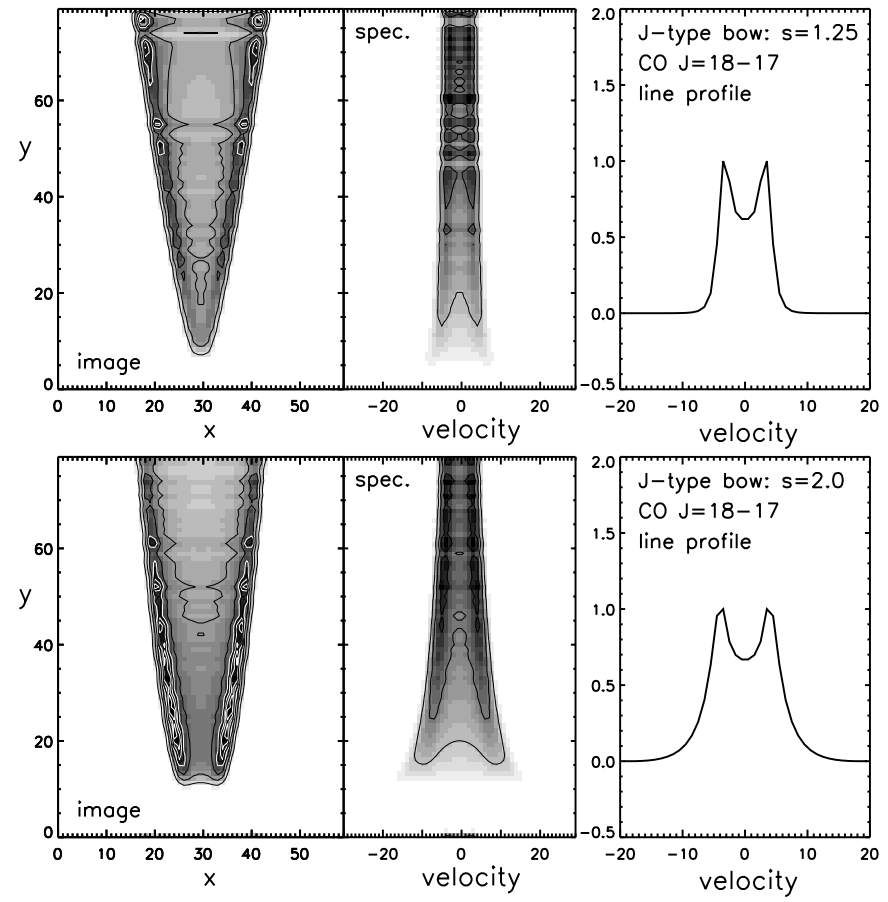

Fig. 12. The flux distributions, velocity-position diagrams and integrated line profiles for the CO $J=18-17$ transition for the following JBOW models. Top: the $s=1.25 J$-type bow shock as analysed in Fig. 7. Bottom: the attempted $s=2.0$ paraboloid fitted to the data in Fig. 6. Both models assume a magnetic field parallel to the bow axis and a bow motion in the plane of the sky. The pixel scale has been reduced in order to include the bulk of the line flux.

very much narrower than both the theoretical maximum for a radiative bow shock (the bow speed, $100 \mathrm{~km} \mathrm{~s}^{-1}$ ) and the maximum for the non-dissociative section of a bow shock (twice the dissociating shock speed, $\sim 50 \mathrm{~km} \mathrm{~s}^{-1}$ ( $J$-type) and $\sim 80-100 \mathrm{~km} \mathrm{~s}^{-1}(C$-type)) (Smith \& Brand 1990). Convolving these line profiles with the instrumental profile, we find no significant increase over the FWHM of the instrumental profile. We conclude that the line widths are consistent with the most plausible bow shock models. The CO line broadening is due to internal motions, ordered (e.g. numerous bullets in a radially expanding wind) or turbulent.

\subsection{Atomic fine structure and $\mathrm{CO}^{+}$lines}

Numerous emission lines from species other than CO and $\mathrm{H}_{2}$ are detected in the ISO data as well. While more open to interpretation (van den Ancker et al. 2000), they can provide some information on the global properties.

The Cepheus A East LWS aperture is close to over a dozen compact HII regions (Hughes 1988). Photodissociation regions (PDRs) exposed to intense farultraviolet flux are characterized not only by high [CII] $158 \mu \mathrm{m}$ fluxes but also by relatively high [FeII] $26 \mu \mathrm{m}$ and $36 \mu \mathrm{m}$ to [CII] $158 \mu \mathrm{m}$ flux ratios (Tielens \& Hollenbach 1985). Hence these [FeII] lines, only detected in Cep A East, may not be shock excited. In contrast, the
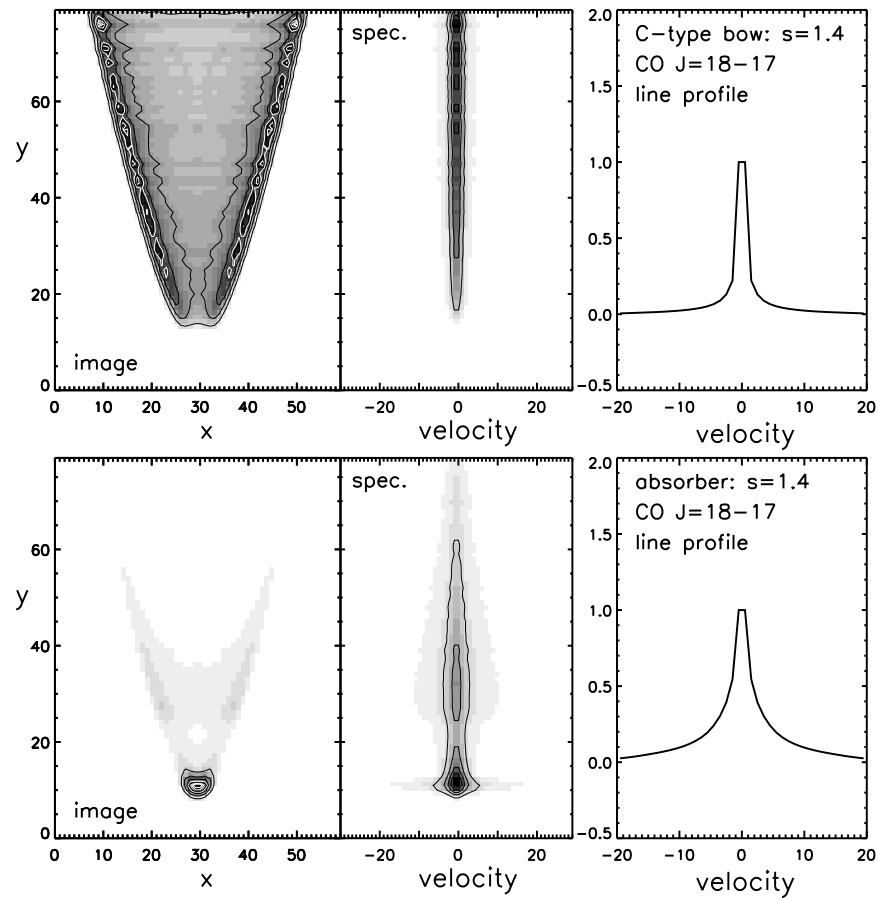

Fig. 13. The flux distributions, velocity-position diagrams and integrated line profiles for the $\mathrm{CO} J=18-17$ transition for the following CBOW models. Top: the $s=1.4 C$-type bow shock as analysed in Fig. 8. Bottom: the $s=1.4$ "Shock Absorber" model fitted to the data in Sect.5.7. Both models assume a magnetic field parallel to the bow axis and a bow motion in the plane of the sky.

fine structure line $[\mathrm{OI}] 63 \mu \mathrm{m}$ is predicted to be strong from both PDRs (Tielens \& Hollenbach 1985) and $C$-shock configurations (Smith 1991). Hence, in Cepheus A East we must consider the observed $63 \mu \mathrm{m}$ to be an upper limit for the shock models. The [SiII] $35 \mu \mathrm{m}$ emission would also be consistent with both PDR and shock predictions whereas strong [SI] $25 \mu \mathrm{m}$ emission arises from dissociative shocks in dense gas $\left(n \geq 10^{5} \mathrm{~cm}^{-3}\right.$; Hollenbach \& McKee 1989) but not from PDRs (Tielens \& Hollenbach 1985). This raises the question of whether the $[\mathrm{SI}]$ emission can be produced from the leading edge of the same $C$-type bow shock which successfully models the molecular emission. We calculate that the dissociative cap of the bow shock is in fact too small to produce significant [SI] $25 \mu \mathrm{m}$ emission but that the $C$-shock itself is likely to be a strong emitter with most of the sulphur remaining in neutral atomic form (Pineau des Forêts et al. 1986).

Three high- $J \mathrm{CO}+$ lines were also detected in Cepheus A East. The origin of the $\mathrm{CO}+$ emission is probably within the cooling layer behind a strong, dissociative $J$-shock located close to the star since then reforming $\mathrm{CO}$ of temperature $\sim 500 \mathrm{~K}$ would be both warm (to generate emission from high rotational levels) and subject to a strong ionising radiation field. $\mathrm{CO}+$ may also arise through collisions between $\mathrm{CO}$ and $\mathrm{H}$ or $\mathrm{H}_{2}$. The activation barrier is, however, high (e.g. Hollenbach \& McKee 1989) and, within a sufficiently strong shock, the CO would already be dissociated. 

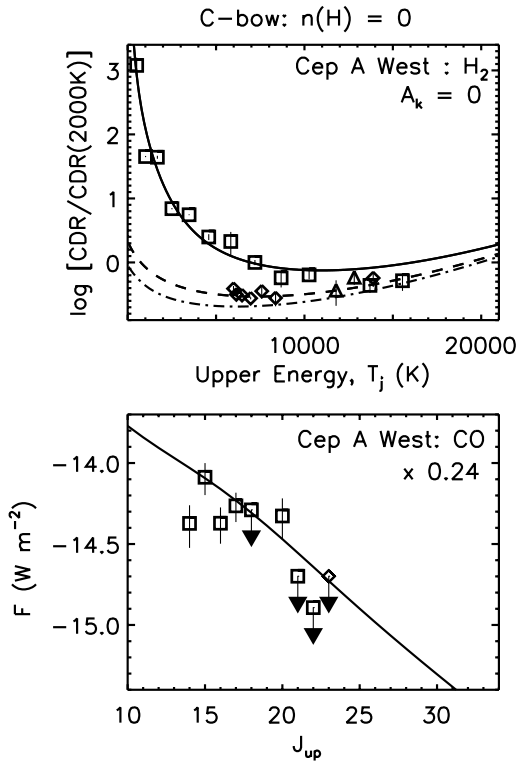

Fig. 14. Model CBOW: a $C$-type bow with speed $100 \mathrm{~km} \mathrm{~s}^{-1}$ and pre-shock density of $3 \times 10^{6} \mathrm{~cm}^{-3}$, ion fraction $2 \times 10^{-7}$ and bow geometry $s=1.4$, are displayed against the $\mathrm{H}_{2}$ (top) and CO (bottom) data for Cepheus A West. An Alfvén speed of $7 \mathrm{~km} \mathrm{~s}^{-1}$ is taken to reduce the [OI] $63 \mu \mathrm{m}$ emission. The oxygen abundance is low $\left(10^{-4}\right)$ and the gas is fully molecular $\left(n\left(\mathrm{H}_{2}\right)=0.5 n\right)$. The full line is the ground vibrational level, and the dashed and dot-dashed lines are the first and second excited levels of $\mathrm{H}_{2}$. The observed $\mathrm{H}_{2}$ columns are displayed as squares (ground), diamonds (first) and triangles (second vibrational level), according to the upper level of the transition.

\section{Interpretation: Cepheus A West}

\section{1. $\mathrm{H}_{2}$ and $\mathrm{CO}$ modelling}

We have compared the various shock models to the complete Cepheus A West data. Wright et al. (1996) presented the $\mathrm{H}_{2}$ data in isolation, concluding that at least two $C$-shocks would be necessary. Smith (2000) has also presented a grid of models for these data which did not include the $0-0 \mathrm{~S}(0)$ line.

Our result is that a high density, fully molecular CBOW model can accurately explain the $\mathrm{H}_{2}$ data, with the first and second vibrational levels being somewhat radiatively depopulated (Fig. 14). There is no evidence here for significant extinction. The $0-0 \mathrm{~S}(1)$ and $0-0 \mathrm{~S}(3)$, however, both lie below the CDR curve, suggesting that, as for the East, the ortho-para ratio in the upstream gas is below 3 . This model requires a boost factor of just 0.24 to be consistent with the CO flux levels. This implies a low $\mathrm{CO}$ abundance of $5 \times 10^{-5}$ and all the $\mathrm{CO}$ to be contained within the SWS beam. Both factors would be surprising. Nevertheless, it is clear from Table 2 that the $\mathrm{CO} / \mathrm{H}_{2}$ flux ratios in Cepheus A West are at least 5 times lower than the values in the East.

A lower density partially atomic $\mathrm{CBOW}$ generates much less CO emission. This low-density model, however, underpopulates the first and second vibrational levels of $\mathrm{H}_{2}$. Hence a second component, a planar $C$-shock with a speed that produces a warm component and a density and atomic hydrogen content which generates an LTE distribution, is then needed. A low density is required for this component so that the contribution to the CO fluxes is negligible.

The structure on the HST infrared and optical images of Cep A is complex (Hartigan et al. 2000). Besides the many bow and arc-shaped structures, there is apparently turbulent shocked gas. In the far-infrared, the turbulent structures might dominate. A few bow shocks along the northern edge possess a stratified chemistry, from an outer $\mathrm{H}_{2}$ bow, to an inner $\mathrm{H} \alpha$ bow, with the [SII] sandwiched inbetween. Hence, we could be observing a magnetic precursor in the form of a bow shock which could mimic a $C$-type bow. The "turbulent" gas, however, is located within the SWS beam and is thus more likely to represent the cool low-density component. Then the hot component could be related to the distinct long linear $\mathrm{H}_{2}$ features in the south of the aperture. High resolution $\mathrm{H}_{2}$ spectroscopy and more sensitive high- $J$ CO measurements $(J>25)$ are needed to distinguish between models.

\subsection{Cepheus $A$ West: Further diagnostics}

The observed flux of the $\mathrm{H}_{2} 0-0 \mathrm{~S}(5)$ line in the SWS aperture is $11.55 \times 10^{-6} \mathrm{~W} \mathrm{~m}^{-2}$. For the high-density $C$-bow model, the predicted bow-averaged surface brightness is $29 \times 10^{-6} \mathrm{~W} \mathrm{~m}^{-2}$. Hence, the surface filling factor in the SWS aperture is 0.4 , the same as derived for Cepheus A East. In the low-density CBOW model, the predicted bowaveraged surface brightness is $6 \times 10^{-6} \mathrm{~W} \mathrm{~m}^{-2}$. The surface filling factor in the SWS aperture is then 2. Both filling factors are roughly consistent with the tangled bow structure of the 1-0 S(1) emission in this region (Hartigan et al. 1996, 2000).

The [OI] $63 \mu \mathrm{m}$ line has an average surface brightness of $1.5 \times 10^{-5} \mathrm{~W} \mathrm{~m}^{-2}$ within the LWS beam. We constrain our models through the intensity ratio of $[\mathrm{OI}](63 \mu \mathrm{m}) / \mathrm{CO}(J=20-19=28.9$ since the two fluxes are measures in the same beam. The model [OI] $(63 \mu \mathrm{m}) / \mathrm{CO}(J=20-19)$ ratios are 27 (high density), 218 (low density) and 42 (the displayed low density, high magnetic field model), both with an oxygen abundance of $10^{-4}$.

Since the CO abundance was found to be low for the high-density CBOW model, the oxygen abundance must similarly be low. One possibility is that the oxygen is tied up in water molecules even before being heated by the shock. However, we can estimate the expected flux in the $\mathrm{H}_{2} \mathrm{O} 126 \mu \mathrm{m}$ line from the tables of Kaufman \& Neufeld (1996). An $\mathrm{H}_{2} \mathrm{O} / \mathrm{CO}$ abundance ratio of about one yields a ratio of $\left(\mathrm{H}_{2} \mathrm{O}(126 \mu \mathrm{m}) / \mathrm{CO}(J=16-15)\right)$ of 0.8 , which is at the limit of the measurement accuracy in that region.

We conclude that we have found a remarkably good single-component fit to the $\mathrm{H}_{2}$ which implies that highdensity $C$-type bow shocks are present. The configuration 


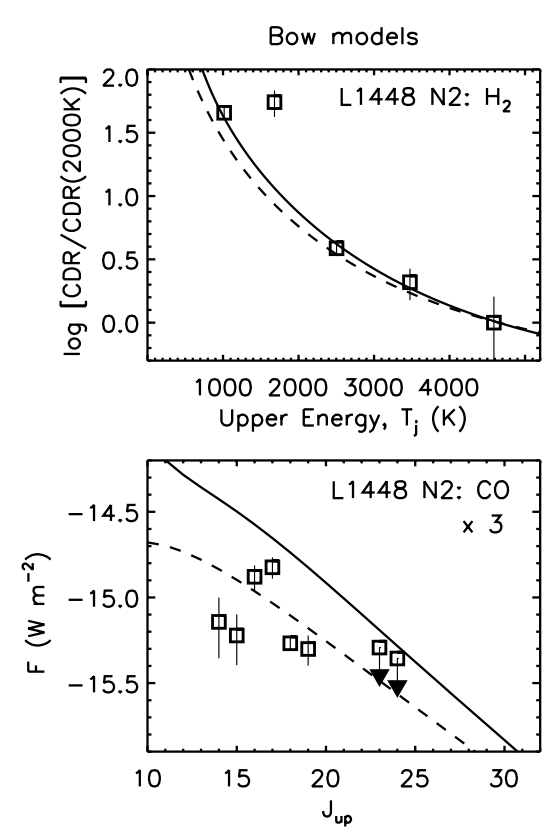

Fig. 15. Models CBOW (full) and JBOW (dotted) plotted against the L $1448 \mathrm{~N} 2$ data. (1) a $C$-type bow with speed $100 \mathrm{~km} \mathrm{~s}^{-1}$ and pre-shock density of $10^{6} \mathrm{~cm}^{-3}$, fully molecular, ion fraction $2 \times 10^{-7}$ and bow geometry $s=1.4$, and (2) a $J$-type bow with pre-shock density of $10^{5} \mathrm{~cm}^{-3}$, fully molecular fraction upstream, speed $80 \mathrm{~km} \mathrm{~s}^{-1}$ and bow shape $s=1.4$, are displayed.

is very similar to that found for the East side, with a bow shape given by $s=1.4$.

\section{Interpretation: L 1448}

Modelling in L 1448 is less conclusive since only a few rotational transitions of $\mathrm{H}_{2}$ per position were detected. We supplement the data with the vibrational excitation as measured in the $K$-band, and indicated by the ratio $R=I(1-0 \mathrm{~S}(1)) / I(2-1 \mathrm{~S}(1))$ for the northern regions by Davis \& Smith (1995). The ratio is constant over the flow from L 1448-mm, with $R=10.4 \pm 2.3$, and constant over a counterflow which appears to stem from L 1448-IRS3 with $R=\sim 20$.

\subsection{1448 N2}

The $\mathrm{H}_{2}$ and $\mathrm{CO}$ in the L 1448 N2 location can be interpreted by both JBOW and CBOW models with longflanked shapes (Fig. 15). The predicted CO excitation and CO flux levels are consistent with the observed data.

An upper limit on the extinction can be set from the $\mathrm{H}_{2}$ 0-0 S(3) line, on assuming the flux is reduced by extrinsic silicate absorption but cannot lie above a linear fit to the rest of the data on the CDR diagram. This yields a very low $2 \mu \mathrm{m}$ extinction of less than 0.4 magnitudes and, indeed, bow shock fits consistent with no extinction.

The $\mathrm{H}_{2}$ ratio $R=I(1-0 \mathrm{~S}(1)) / I(2-1 \mathrm{~S}(1))$ observed by Davis \& Smith (1995) for the N2 region is 13.5 (taking the flux-weighted average of the knots). This is consistent with the model value of 11.5 (CBOW) but compares less well with 17.6 (JBOW). A low density bow with pre-shock density of $10^{5} \mathrm{~cm}^{-3}$ also fits the $\mathrm{H}_{2}$ and $\mathrm{CO}$ diagrams with bow shape $s=1.2$, but the predicted ratio $R=36.4$ is then too large.

Extraordinary low levels of [OI] $63 \mu \mathrm{m}$ emission are found in $\mathrm{L} 1448$. This is inconsistent with the cool $\mathrm{H}_{2}$ spectra. The J-bow model, as for Cep A, predicts at least an order of magnitude too much [OI] $63 \mu \mathrm{m}$ emission, generated in the long cool bow tails. The $C$-bow model yields a $[\mathrm{OI}](63 \mu \mathrm{m}) / \mathrm{CO}(J=17-16)$ flux ratio of 73 , even with the low oxygen abundance of $10^{-4}$, still a factor of 17 larger than observed.

One solution would be that the pre-shock chemistry in our model is incorrect: the oxygen is, instead, tied up in $\mathrm{H}_{2} \mathrm{O}$ or other species. This may be feasible if the pre-shock gas is warm and the atomic hydrogen fraction is very low, both factors which reduce the atomic oxygen abundance when the oxygen chemistry is in equilibrium. This argument is supported by the global structure of the outflow as a parsec-scale molecular flow (Eislöffel 2000), in which the gas entering shock N2 has been preprocessed through previous shocks converting the atomic oxygen into $\mathrm{H}_{2} \mathrm{O}$.

A more likely solution is however that the oxygen fine-structure lines are optically thick. The modelled [OI] $63 \mu \mathrm{m}$ to [OI] $145 \mu \mathrm{m}$ flux ratio is 112 , an order of magnitude larger than observed, evidence that the $63 \mu \mathrm{m}$ line is optically thick. Hollenbach \& McKee (1989) state a column density of $4.9 \times 10^{20} \mathrm{~cm}^{-2}$ would provide an optical depth of unity at the line centre for this transition. We find that the column of cool gas in the wings of the $C$-type bows is typically $2-4 \times 10^{21} \mathrm{~cm}^{-2}$.

The predicted surface filling factor of the $\mathrm{H}_{2}$ emission is low. This problem arises from the low observed fluxes in comparison to Cepheus A. For the L 1448 N2 location, the $0-0 \mathrm{~S}(5)$ surface brightness in the SWS aperture is $10^{-6} \mathrm{~W} \mathrm{~m}^{-2}$. The CBOW model predicts a bow-average surface brightness of $5.8 \times 10^{-5} \mathrm{~W} \mathrm{~m}^{-2}$, which implies an aperture filling factor of 0.02 . This is rather low although the 1-0 S(1) emission certainly does not fill the SWS aperture. A solution investigated here is that the hydrogen in the outflow is predominantly atomic. Then, the powerful shocks would generate less $\mathrm{H}_{2}$ emission. We also find that a lower density is then permissible in the excitation modelling. We thus arrive at a model with just ten per cent of the atoms tied up in molecular hydrogen, and a pre-shock density of $3 \times 10^{5} \mathrm{~cm}^{-3}$. The $0-0 \mathrm{~S}(5) \mathrm{SWS}$ filling factor is then 0.4 and the CO boost factor is 18 . The atoms would reform on grains in a time of order $3 \times 10^{16} / n=3000 \mathrm{yr}$, assuming standard grain properties. This model, however, predicts high intrinsic oxygen $63 \mu \mathrm{m}$ emission since the high atomic fraction would ensure that $\mathrm{H}_{2} \mathrm{O}$ and $\mathrm{OH}$ are dissociated by collisions with $\mathrm{H}$, producing abundant atomic oxygen.

There is quite uniform [CII] emission across L 1448 . This is not expected to arise directly from the outflows but is consistent with excitation by the local radiation field (see Nisini et al. 1999 for further discussion). 


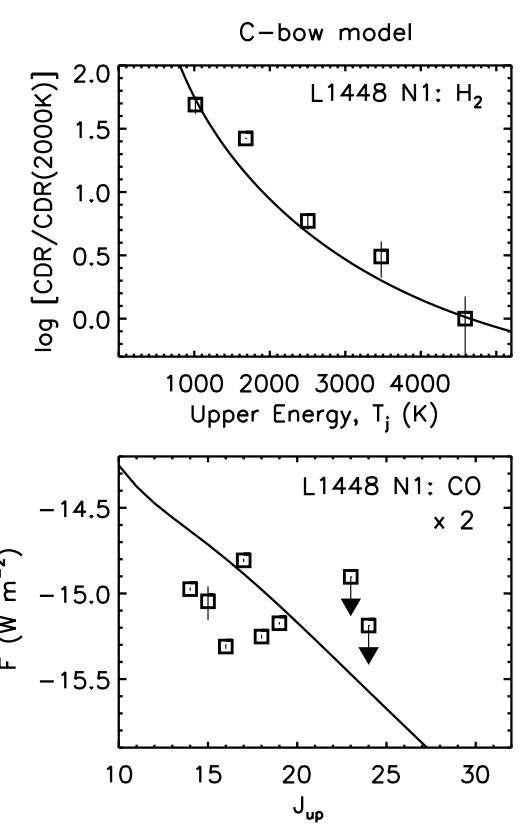

Fig. 16. The L $1448 \mathrm{~N} 1$ data with the CBOW model displayed for N2 but with $s=1.35$.

\section{2. $L 1448 N 1$}

The $\mathrm{H}_{2}$ rotational excitation is even lower in the N1 location. We find a CBOW model with $s=1.35$ and pre-shock density $10^{6} \mathrm{~cm}^{-3}$ fits the data (Fig. 16). This predicts $R=$ 13.4 which is somewhat high. We also find that a higher density reproduces the observed $1-0 / 2-1$ ratio but that this density then overestimates the $\mathrm{CO}$ flux level. A lower density has the opposite result. The $[\mathrm{OI}](63 \mu \mathrm{m}) / \mathrm{CO}(J=$ 17-16) flux ratio is 140 for $\mathrm{O}$ and $\mathrm{CO}$ abundances both of $2 \times 10^{-4}$, again requiring the oxygen to be tied up in molecules or dust or that the line is optically thick. The modelled [OI] $63 \mu \mathrm{m}$ to [OI] $145 \mu \mathrm{m}$ flux ratio is 112 , an order of magnitude larger than observed. This suggests that the $63 \mu \mathrm{m}$ line is optically thick.

\section{3. $L 1448 C$}

The central region cannot be reliably analysed since the $\mathrm{H}_{2}$ data we have are unsatisfactory. Nisini et al. (1999), however, have published upper limits for a few other lines which we include in our modelling. This clearly corroborates that an excitation of the $\mathrm{H}_{2}$ rotational spectra in this region is very similar to N2 and N1 (Fig. 17).

The water lines are difficult to use as shock diagnostics (e.g. Nisini et al. 1999). We have used the Tables and Figures provided by Kaufman \& Neufeld (1996) to predict fluxes from the CBOW models in some detail. We find that the observed ratio of $4_{14}-3_{03} / 0-0 \mathrm{~S}(2)$ is consistent although the data are inadequate for a detailed test.

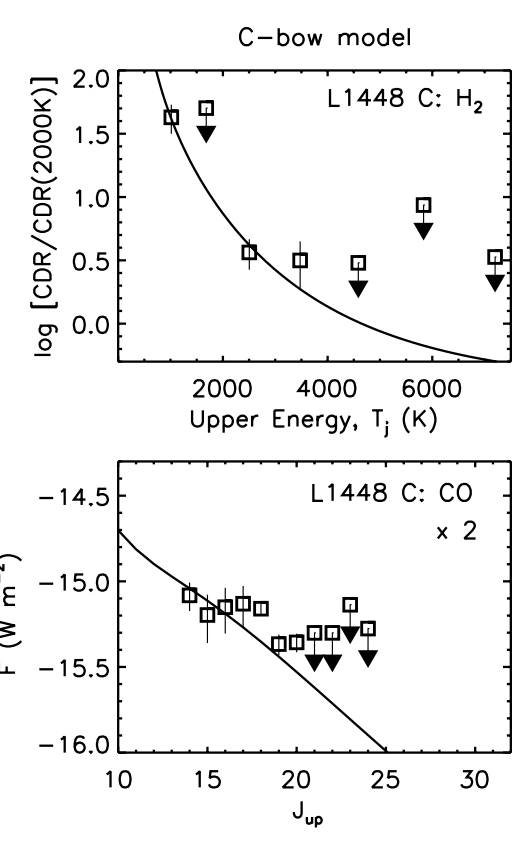

Fig. 17. Model CBOW plotted against the L $1448 \mathrm{C}$ data. The $C$-type bow applied to N2 is taken here, with a lower CO boost factor providing a consistent fit.

\subsection{1448 South}

We do not have comprehensive data sets for the areas south of the source. For the S1 location, we take the same models as for the central location, and check their consistency with the available data. This then predicts the $0-0 \mathrm{~S}(5)$ flux of $8 \times 10^{-16} \mathrm{~W} \mathrm{~m}^{-2}$. We take the analysis of S1 no further here.

Heading south, in Fig. 18 we show that shock configurations are again necessary to explain the $\mathrm{H}_{2}$ data for location S2 (the S3 region is only slightly warmer). We find: (1) the $\mathrm{H}_{2}$ excitation appears very similar to the Northern locations but (2) the CO emission is considerably less. The obvious implication of lower CO fluxes is that the density is lower. However, the $\mathrm{H}_{2}$ line strengths are comparable to those in the North of the outflow. Moreover, a lower density would alter the predicted shock ensemble: we find that a $C$-type bow with $s=1.2$ (dot-dashed lines) would be necessary with $n=10^{5} \mathrm{~cm}^{-3}$. This would make the fact that the excitation appears similar as in the North as pure coincidence. An alternative answer would be that extinction has decreased the apparent excitation. We find, however, that the $0-0 \mathrm{~S}(3)$ flux is not sufficiently depressed to allow for much extinction.

The answer we favour is that the $\mathrm{CO}$ abundance is low, with CO frozen out onto grains. Then, all other parameters employed for the N2 CBOW model remain unchanged, as shown by the solid-line fit displayed in Fig. 18.

\section{Implications and conclusions}

Our main result is that we can model the ISO data of Cep A and L 1448 with a broad distribution of shock 

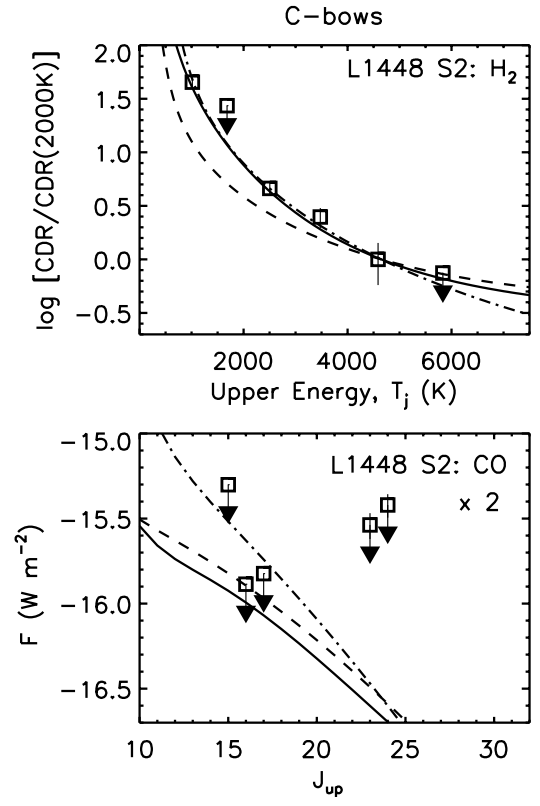

Fig. 18. Models CBOW with bow shape 1.4 (dashed) along with the data from the $\mathrm{S} 2$ position of L 1448. Bow speed $100 \mathrm{~km} \mathrm{~s}^{-1}$, pre-shock density of $10^{6} \mathrm{~cm}^{-3}$, molecular fraction of $0.5 \mathrm{n}$, ion fraction $2 \times 10^{-7}$, and $\mathrm{CO}$ and $\mathrm{O}$ abundances both $1 \times 10^{-5}$. Two lower density models are also shown with $s=1.2$ (dot-dash) and $s=1.4$ (dashed), for a density of $10^{5} \mathrm{~cm}^{-3}$, but a standard $\mathrm{CO}$ abundance of $2 \times 10^{-4}$.

strengths, as typified by bow shocks with long flanks. The implied ensembles of shocks are remarkably similar. $\mathrm{CO}$ and $\mathrm{O}$ abundances in the range $2-4 \times 10^{-4}$ and $0.5-1 \times 10^{-4}$ are predicted, with the $63 \mu \mathrm{m}$ oxygen finestructure line optically thick.

This study emphasizes the future need for comparable apertures across the infrared so that we can model the abundances with more confidence. A higher sensitivity for CO rotational levels above $J=20$ would aid the interpretation. Simultaneous $K$-band and $H$-band spectra would also provide the type of constraint which would reveal the shock physics. ISO had a very extensive programme of observations, making it impossible to carry out measurements also achievable from the ground.

\subsection{Cepheus $A$}

Shock configurations with a strong weighting towards the weaker shocks are necessary to explain the $\mathrm{H}_{2}$ excitation.

$C$-type physics provides the best interpretation of the low excitation, the $\mathrm{CO}$ high- $J$ fluxes and the [OI] $63 \mu \mathrm{m}$ fine-structure emission line.

The two lobes, although far apart, and dynamically distinct, both contain high density shocked gas $(1-3 \times$ $\left.10^{6} \mathrm{~cm}^{-3}\right)$, both with the same very low overall excitation. Such low excitation has been found previously for the Cepheus E outflow (Eislöffel et al. 1996) from the vibrational $\mathrm{H}_{2}$ lines, and also for DR 21 (Smith et al. 1998) from $\mathrm{SWS}$ rotational $\mathrm{H}_{2}$ line data. The low excitation thus appears quite common in the high-powered outflows (with
OMC-1, where a paraboloidal bow $(s=2)$ provides a fit, as the exception).

The inferred bow shocks are of the shape $s \sim 1.4$. High resolution observations reveal a mixed $\mathrm{H}_{2}$ bag in Cepheus A West: numerous linear features and clumps, especially in the south and centre, bow shocks to the west and magnetic precursors to the north (Hartigan et al. 2000).

The energetics of outflows can be determined from ISO data without the uncertainties introduced by extinction. The $C$-type bow shock models yield the total radiated luminosity from the $C$-type sections. For the most plausible models we calculate total shock luminosities of $52.8 L_{\odot}$ and $61.6 L_{\odot}$ for Cepheus A East and West from within the LWS apertures (assuming a distance of $725 \mathrm{pc}$ ). This compares to a mechanical luminosity of $37 L_{\odot}$ for the bipolar outflow derived from CO observations (Narayanan \& Walker 1996). This suggests that the outflow is still being strongly driven by the shocks. When the energy source weakens, we expect the driving shocks to rapidly fade while the outflow switches from a driven into a coasting mode.

The predicted width of both $\mathrm{CO}$ and $\mathrm{H}_{2}$ lines from bow shocks are narrow, even in high magnetic field models $\left(F W H M<10 \mathrm{kms}^{-1}\right.$, see Sect. 5.12). Hence, if wide lines were detected, other larger internal motions must be dominant. Then, intrinsic line profiles are probably very similar in shape and width from all transitions, as apparent in Cep A East (Fig. 3).

The total radiation and mechanical luminosity is only a small fraction of the luminosity generated by the central group of hot stars, estimated to be $1.4 \times 10^{4} L_{\odot}$. Hence over $1 \%$ must be channeled into the outflow. If this outflow is to be driven by the momentum of the radiation then it must have a speed in excess of $0.01 c$. Therefore, we conclude that the outflow is not radiatively driven.

Our SWS aperture covers the central turbulent region in the west. Indeed, a model for decaying supersonic turbulence is a viable explanation. However, one must catch the decay at a specific time to provide a fit. Will a timeaveraged decaying turbulent field yield an interpretation? Integrating the turbulent spectra over time yields a high excitation prediction: the emission is dominated by the strong shocks which form early in the flow. This then implies that the injected shock spectrum itself is responsible for what is observed.

Turbulence is inevitably created behind bow shocks: a curved shock generates vorticity and supersonic vorticity dissipates in shocks. Smith (1995) estimated that a sizeable fraction of the available energy of a bow shock is dissipated in this manner, and this energy would be channeled into low velocity shocks within the wake.

\section{2. $L 1448$}

L 1448 possesses no significant spatial variation in $\mathrm{H}_{2}$ excitation. We find $s \sim 1.3-1.4$ for $C$-type bows. 
Densities or filling factors are a factor of 10 lower than in Cep A. The gas is not fully molecular. Then (1) $\mathrm{H}-\mathrm{H}_{2}$ collisions maintain high $2-1 / 1-0 \mathrm{~K}$-band ratios, as observed, and (2) the $\mathrm{CO} / \mathrm{H}_{2}$ line ratios are boosted in the models without an excessively high $\mathrm{CO} / \mathrm{H}$ abundance (see also Nisini et al. 1999).

The spatially turbulent region of the southern lobe is characterised by very low $\mathrm{CO} / \mathrm{H} 2$ flux ratios. The data here is of poor quality. If confirmed, then we suggest that this is most likely due to $\mathrm{CO}$ depletion onto grains.

The lower density in L 1448 is consistent with the lower power of the outflow and luminosity of the protostar. For the N1 beam, we calculate a total shocked luminosity of $1.5 L_{\odot}$, and estimate a total shocked output from L 1448 of $\sim 6 L_{\odot}$. The outflow mechanical luminosity is estimated at $6.5 L_{\odot}$ (Barsony et al. 1998). It is most plausible that the mechanical and radiated luminosities should again be equal since L 1448 is a Class 0 protostar: the driving jets should be at their strongest (Smith 2000) and the energies which are transferred into heat and bulk motion are roughly equal in a shock.

The total power radiated in the molecular lines over the whole outflow is estimated from the overlapping LWS beams. Most of the radiation is predicted to be emitted in the mid- $J$ CO lines originating from the $J=3$ to the $J=10$ levels (independent of the particular model). These lines were not observed by ISO. The total radiated power for the L 1448 models is $\sim 0.9 L_{\odot}$, about $10 \%$ of the bolometric luminosity of the driving protostar $\left(9 L_{\odot}\right.$, Barsony et al. 1998). The mechanical luminosity is $\sim 3 L_{\odot}$. In a momentum-driven outflow, one expects that the shocked emission would equal the mechanical power supplied to the environment since the driving shocks are dissipative. We suggest that the protostar accretes non-uniformly, and hence ejects material non-uniformly. At present, the protostar is inbetween such pulsations. Then, during active phases the bolometric luminosity, followed by the shocked luminosity, could increase by a factor of perhaps 10 . The mechanical luminosity, on the other hand, varies little since it is the power averaged over the outflow lifetime. That the ejections are pulsed is supported by the presence of CO bullets (Bachiller et al. 1998). It should be remarked, however, that extended dust emission associated with the outflow has a luminosity $\sim 6 L_{\odot}$. Hence, the estimates for either the mechanical or shock powers may be in error.

Acknowledgements. Jochen Eislöffel and Dirk Froebrich received financial support from the DLR through Verbundforschung grant 50 OR 99049.

We thank Eckhard Sturm from the ISO Spectrometer Data Centre at MPE for help with the data reduction of the ISO spectra.

The ISO Spectral Analysis Package (ISAP) is a joint development by the LWS and SWS Instrument Teams and Data Centers. Contributing institutes are CESR, IAS, IPAC, MPE, RAL and SRON.

LIA is a joint developement of the ISO-LWS Instrument Team at Rutherford Appleton Laboratories (RAL, UK - the PI
Institute) and the Infrared Processing and Analysis Center (IPAC/Caltech, USA).

\section{References}

Bachiller, R., Martin-Pintado, J., Tafalla, M., Cernicharo, J., \& Lazareff, S. 1990, A\&A, 231, 174

Barsony, M., Ward-Thompson, D., André, P., \& O'Linger, J. 1998, ApJ, 509, 733

Black, J. H., \& van Dishoeck, E. F. 1987, ApJ, 322, 412

Brand, P. W. J. L., Moorhouse, A., Burton, M. G., et al. 1988, ApJL, 334, 103

Burton, M. G., \& Haas, M. R. 1997, A\&A, 327, 309

Burton, M. G., Hollenbach, D. J., \& Tielens, A. G. G. 1992, ApJ, 399, 563

Caux, E., Ceccarelli, C., Castets, A., et al. 1999, A\&A, 347, L1

Clegg, P. E., Ade, P. A. R., Armand, C., et al. 1996, A\&A, 315, L38

Davis, C. J., \& Smith, M. D. 1995, ApJ, 443, L41

de Graauw, T., Haser, L. N., Beintema, D. A., et al. 1996, A\&A, 315, L49

Di Giorgio, A. M., et al. 1999, in Optical and Infrared Spectroscopy of Circumstellar Matter, ASP Conf. Ser., 188, 93

Draine, B. T., Roberge, W. G., \& Dalgarno, A. 1983, ApJ, 264, 485

Eislöffel, J., Smith, M. D., Davis, C. J., \& Ray, T. P. 1996, AJ, 112, 2086

Eislöffel, J. 2000, A\&A, 354, 236

Eislöffel, J., Smith, M. D., \& Davis, C. J. 2000, A\&A, 359, 1147

Everett, M. E., DePoy, D. L., \& Pogge, R. W. 1995, AJ, 110, 1295

Fernandes, A. J. L., \& Brand, P. W. J. L. 1998, Ap\&SS, 261, 179

Fernandes, A. J. L., \& Brand, P. W. J. L. 1995, MNRAS, 274, 639

Gómez, J. F., Sargent, A. I., Torrelles, J. M., et al. 1999, ApJ, 514,287

Hartigan, P., Carpenter, J. M., Dougados, C., \& Skrutskie, M. F. 1996, AJ, 111, 2470

Hartigan, P., Morse, J., Palunas, P., Bally, J., \& Devine, D. 2000, AJ, 119, 1872

Hollenbach, D. J., \& McKee, C. F. 1989, ApJ, 342, 306

Hughes, V. A. 1988, ApJ, 333, 788

Kaufman, M. J., \& Neufeld, D. A. 1996, ApJ, 456, 611

Kessler, M. F., Steinz, J. A., Anderegg, M. E., et al. 1996, A\&A, 315, L27

Le Bourlot, J., Pineau Des Forêts, G., \& Flower, D. R. 1999, MNRAS, 305, 802

Mac Low, M. M., \& Smith, M. D. 1997, ApJ, 491, 596

McCartney, M. S. K., Brand, P. W. J. L., Burton, M. G., \& Chrysostomou, A. 1999, MNRAS, 307, 315

McKee, C. F., Storey, J. W. V., Watson, D. M., \& Green, S. 1982, ApJ, 259, 647

Narayanan, G., \& Walker, C. K. 1996, ApJ, 466, 844

Neufeld, D. A., \& Kaufman, M. J. 1993, ApJ, 418, 263

Nisini, B., Benedettini, M., Giannini, T., et al. 1999, A\&A, 350,529

Nisini, B., Benedettini, M., Giannini, T., et al., 2000, A\&A, 360,297 
O`Linger, J., Wolf-Chase, G., Barsony, M., \& Ward- Smith, M. D., Davis, C. J., \& Lioure, A. 1997, A\&A, 327, 1206 Thompson, D. 1999, ApJ, 515, 696

Smith, M. D., \& Mac Low, M. M. 1997, A\&A, 326, 801

Pineau des Forêts, G., Roueff, E., \& Flower, D. 1986, MNRAS, Smith, M. D., Mac Low, M. M., \& Zuev, J. 2000, A\&A, 356, 223,743

Smith, M. D. 1986, MNRAS, 223, 57

Smith, M. D. 1991, MNRAS, 253, 175

Smith, M. D. 1994a, MNRAS, 266, 238

Smith, M. D. 1994b, MNRAS, 289, 256

Smith, M. D. 1995, A\&A, 296, 789

Smith, M. D. 2000, IAJ, 27, 37

Smith, M. D., \& Brand, P. W. J. L. 1990, MNRAS, 245, 108

Smith, M. D., Brand, P. W. J. L., \& Moorhouse, A. 1991a, MNRAS, 248, 451

Smith, M. D., Brand, P. W. J. L., \& Moorhouse, A. 1991b, MNRAS, 248, 730

Smith, M. D., Eislöffel, J., \& Davis, C. J. 1998, MNRAS, 297, 287

Swinyard, B. M., Clegg, P. E., Ade, P. A. R, et al. 1996, A\&A, $315, \mathrm{~L} 43$

Tielens, A. G. G. M., \& Hollenbach, D. 1985, ApJ, 291, 722

van den Ancker, M. E., Tielens, A. G. G. M., \& Wesselius, P. R. 2000, A\&A, 358, 1035

Vastel, C., Caux, E., Ceccarelli, C., et al. 2000, A\&A, 357, 994

Wardle, M. 1990, MNRAS, 246, 98

Whittet, D. C. B., Bode, M. F., Longmore, A. J., et al. 1988, MNRAS, 233, 321

Wright, C. M., Drapatz, S., Timmerman, R., et al. 1996, A\&A, 315, L301 687 